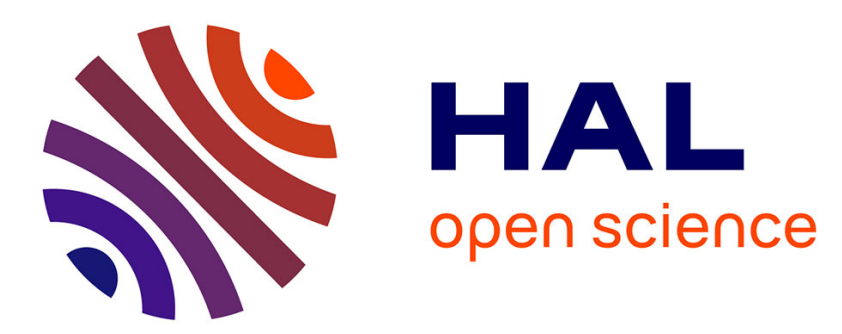

\title{
A new PDE-based approach for singularity-preserving regularization: application to degraded characters restoration
}

Fadoua Drira, Frank Le Bourgeois, Hubert Emptoz

\section{- To cite this version:}

Fadoua Drira, Frank Le Bourgeois, Hubert Emptoz. A new PDE-based approach for singularitypreserving regularization: application to degraded characters restoration. International Journal on Document Analysis and Recognition, 2011, 3, 15, pp.183-212. 10.1007/s10032-011-0165-5 . hal01354467

\section{HAL Id: hal-01354467 \\ https://hal.science/hal-01354467}

Submitted on 7 Mar 2017

HAL is a multi-disciplinary open access archive for the deposit and dissemination of scientific research documents, whether they are published or not. The documents may come from teaching and research institutions in France or abroad, or from public or private research centers.
L'archive ouverte pluridisciplinaire HAL, est destinée au dépôt et à la diffusion de documents scientifiques de niveau recherche, publiés ou non, émanant des établissements d'enseignement et de recherche français ou étrangers, des laboratoires publics ou privés. 


\section{A New PDE-based Approach for Singularity-Preserving Regularization: Application to Degraded Characters Restoration}

Received: 10 Mars 2010 / Accepted: date

\begin{abstract}
The massive digitization of heritage documents have raised new prospects for Research like degraded document image restoration. Degradations harm the legibility of the digitized documents and limit their processings.

As a solution, we propose to tackle the problem of degraded text characters with PDE (Partial Differential Equation)-based approaches. Existing PDE-approaches do not preserve singularities and edge continuities while smoothing. Hence, we propose a new anisotropic diffusion by adding new constraints to the Weickert coherence enhancing diffusion filter in order to control the diffusion process and to eliminate the inherent corner rounding. A qualitative improvement in the singularity preservation is thus achieved. Experiments conducted on degraded document images illustrate the effectiveness of the proposed method compared to another anisotropic diffusion approaches. We illustrate the performance with the study of the optical recognition accuracy rates.
\end{abstract}

Keywords degraded text characters · document images · PDE-based approaches - optical character recognition $\cdot$ restoration $\cdot$ reconstruction $\cdot$ enhancement

\section{Introduction}

Historical documents are of great interest to human beings. Nowadays, recent techniques help in producing digital copies of these documents to provide wider use of its rich resources while preserving rare, valuable and fragile documents. Nevertheless, the quality of these digital copies depends greatly on the quality of the original

REGIM - ENIS, Route de Soukra, km 4 w.3038 Sfax, Tunisie E-mail: fadoua.drira@ieee.org

LIRIS - INSA Lyon, 20 avenue Albert Einstein, 69621 Villeurbanne Cedex, France

E-mail: Franck.lebourgeois@insa-lyon.fr

E-mail: hubert.emptoz@liris.cnrs.fr documents. These are often affected by several types of degradations limiting their use [1]. For instance, a poor text quality reduces the performance of the Optical character recognition (OCR), a useful tool mainly in digitalizing libraries and in computer-assisted indexing [2]. That is why we need document restoration methods in order to improve the OCR accuracy and the quality of the digital copy of the original degraded document image. Restoring could thus enhance human readability and allows further application of image processing techniques such as segmentation and character recognition.

Indeed, in earlier applications, image restoration has often been used to denoise (noise is due to the electronic, the CCD sensor and the image preprocessing, quantification and compression processes) or deblur (blur is due to long exposition, poor focusing, out-of-focus cameras, camera or object motion during exposure) the image. For instance, image noise/blur is stemmed from the image acquisition process that results in pixel values that do not reflect the true intensities of the real scene or the original support. Noise or blur could be then introduced into an image, depending on how the image is created. Classic image denoising algorithms look for removing additive white stationary Gaussian noise, while preserving the original image details. Examples of such algorithms are Gaussian smoothing [3], anisotropic diffusion, total variation [4], non-local means algorithm [5, 6] followed by other improvement for speeding up the original filter $[7,8]$ and for allowing more flexibility $[9$, 10]. Buades et al [5] pointed out that non-local means filter is a special case of bilateral filter which gives better edge preserved denoising results. The concept of the exemplar-based approaches was also successfully introduced in image denoising [11] and image deblurring [12]. The Field of Experts (FoE) model is another interesting approach recently used in image denoising [13]. By introducing this model, classical Markov random field (MRF) models becomes more powerful. All of the previously mentioned approaches use the natural redundancy of information in images to remove the noise. We re- 

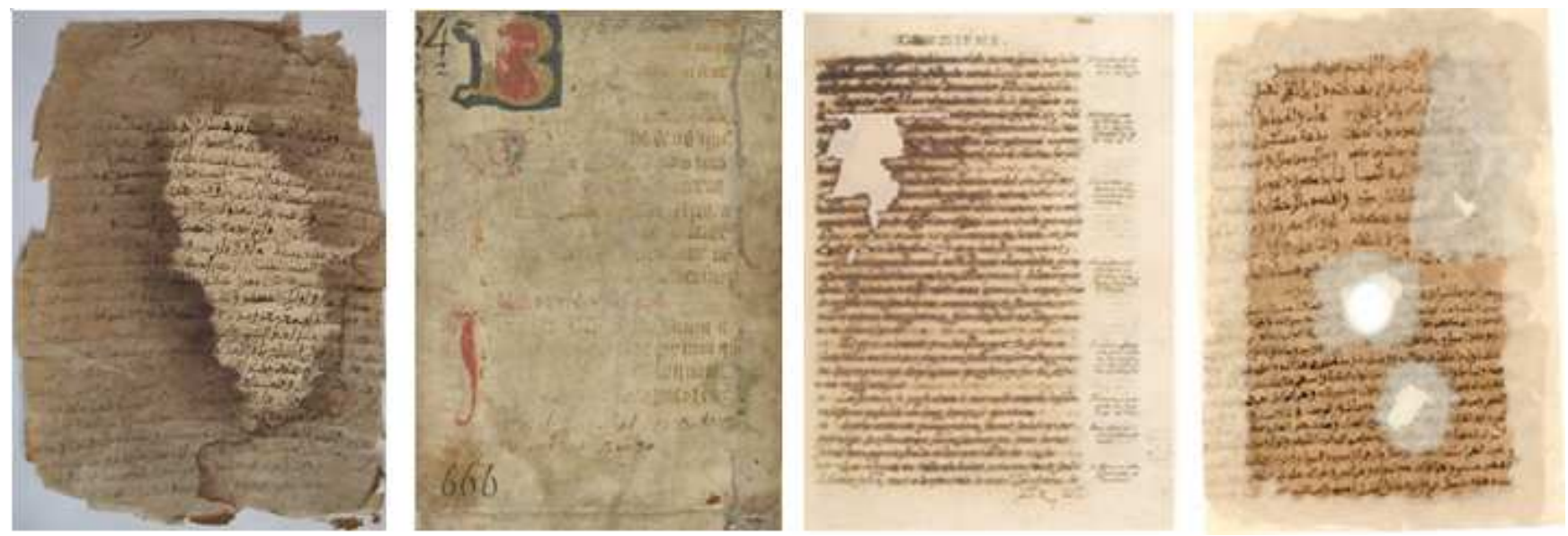

Fig. 1 Illustration of some examples of degraded document images.

fer the reader to [14] for the state-of-the-art denoising results. The application area of restoration includes for example image surveillance and medical images where the imaging system introduces a light distortion. Most of these works model a degraded image as an original image that has been subject to linear frequency distortion and additive noise injection. These two sources of degradations are the most studied.

Faced with old documents, other types of degradations, such as the mixture of information (ink bleedthrough, spots), loss of information (gaps, discontinuities), pattern deformation (geometric deformation, noise around contours, unwanted jagged edges), appear leading to other restoration techniques adapted to them. In fact, document images with common noise models can be enhanced using traditional image restoration techniques such as Median filtering, Weiner filtering [15], morphological filters [16,17], multi-resolution pyramid and fuzzy edge detectors [18], etc. Nevertheless, in practice, these popular models are not well suited to degradations arising from phenomena such as document aging or ink bleeding. Specific degradation models for both document [19] and text [20,21] are thus introduced. For reconstructing low-resolution text image, H. Luong et al. [22] exploit the repetitive behaviour of the characters on the whole text image and not only on a local surrounding neighbourhood. J. Banerjee et al. [23] introduce a probabilistic context model using an MRF to deal with severe document degradations including cuts, blobs, merges and vandalized documents. The loss of information is often repaired by diffusion approaches like inpainting and anisotropic diffusion. The mixture of information is generally restored by the separation of sources (ICA [24], Markov model [25], diffusion [26,27], classification or segmentation techniques $[28,29]$. For pattern deformations, we can use geometric restoration, mathematical morphology [30] and even diffusion processes.
The main topic of this study is restoring degraded text character. The latter consists of repairing the shapes of the features as well as extrapolating lost information. Text restoration remains a challenge due to the variety of fonts, colors and even to the presence of complex backgrounds [28]. Moreover, text characters degradations are characterized by a random loss of information which makes their treatment very difficult to deal with. It has been noticed that the state-of-the-art of the main works dealing with textual document restoration is very limited. Few algorithms have been developed by the community [31-35]. Most of them give interesting results; however, their performance relies upon an efficient character segmentation and/or recognition which limits their application. For instance, applying machine learning methods for text restoration encounters difficulties caused by character size and grayscale variations. They are also not generic enough to treat any document images since they are designed in advance for a specific content. Textual document images' restoration is an important topic and many projects are proposed in order to find solutions. We can cite as an example the European project IMPACT [36], led by IBM and started in 2009, a project that has an important workpackage on image restoration to improve OCR result.

This paper proposes as a solution to textual document image restoration the application of Partial Diffusion Equation (PDE) - based techniques, known as an effective way to restore natural degraded images. Based on a study done on image restoration techniques and attracted with its useful properties, we propose testing the efficiency of PDE-based approaches for restoring degraded characters as a first step. As a second step, we propose a new PDE-based approach which preserves the character structures and enhances the coherency of the strokes. We must notice that enhancing text in degraded document images using PDE-based approaches has been already introduced in the literature but our main con- 
tribution is twofold. We test the application of the most efficient PDE-based approaches on degraded document and we propose a new approach that deals successfully with singularity-preserving regularization, a major characteristic of textual document images. Diffusion process, a common framework for the main type of document image degradations, justifies our choice for taking into account such process.

After a brief study of document image degradations in Section 2, we will introduce some PDE - based approaches in section 3 . In addition, we will highlight, through experimental results, the advantages and shortcomings of each of them. We refer the reader [37-40] for a more detailed description of a large number of PDE-based approaches. This study proves that a specific class of these approaches, called "matrix-valued diffusivity", is wellsuited for the processing of oriented features. Nevertheless, none of them preserves singularities as important features in textual documents. This problem represents the motivation of the next section (Section 4) where we propose a new PDE-based approach. This proposition tends to preserve discontinuities as well as singularities of the image while being smoothed. Some experiments done on degraded document images indicate, in section 5 , that our proposition provides an efficient tool for denoising, reconstructing and even restoring resized images (case of magnification). This leads to a noticeable improvement of the OCR system's accuracy proven through the comparison of the OCR recognition rates before and after the diffusion process. A comparative study with results generated after applying other PDE-based approaches is also given.

\section{A study of document image degradations}

\subsection{Problem Statement}

The term degradation means, as suggested by Henry S. Baird [33], "every sort of less-than ideal properties of real document images". In fact, document image degradations could be divided into different sets according to their origin. We can distinguish, for example, defects stemmed from time effect as well as digitization. By time effect defects, we refer to all defects related to bad environmental conditions mainly the humidity caused by an unfitted storage during many years. We notice that once the old document is scanned, the previous defects become a part of the digitized image document. During the scanning process, other types of degradations could be added. For instance, digital degradations are generated through the use of some image processing techniques (high compression rate, low resolution, binarization,...). Mechanical degradations refer to bad digitizing conditions, defected material, low scanner performance,...
The digitization process could be "direct" if it is done from the original document or "indirect" in the case of using a copy (microfilm, negatives, photography, printed documents...). It is obvious that "direct" digitization is more complicated than "indirect" digitization but generates images with better qualities. Examples of degraded document images are given in figure 1 . These images available within our team were provided by the French Institute of Research on Text History, the archive of "Chatillon-Chalaronne" and the French National Biblio.

In order to deal with virtual restoration, [1] presents a typology of document image degradations defined according to the further treatment that will be undertaken. This typology is thus intended to image processing. It is decomposed into two classes: uniform and nonuniform degradations. Uniform degradations affects the entire document. This could be illustrated by geometrical degradation and non-uniform illumination which are common phenomena in document analysis resulting from the scanning of thick documents. Non-uniform degradations affects local parts in the document. Degradation as such could affect either the paper support (noisy background) or the letters (noisy foreground). Hence, the present work focuses on noisy foreground degradations (Fig.2) which are the major causes of OCR errors.

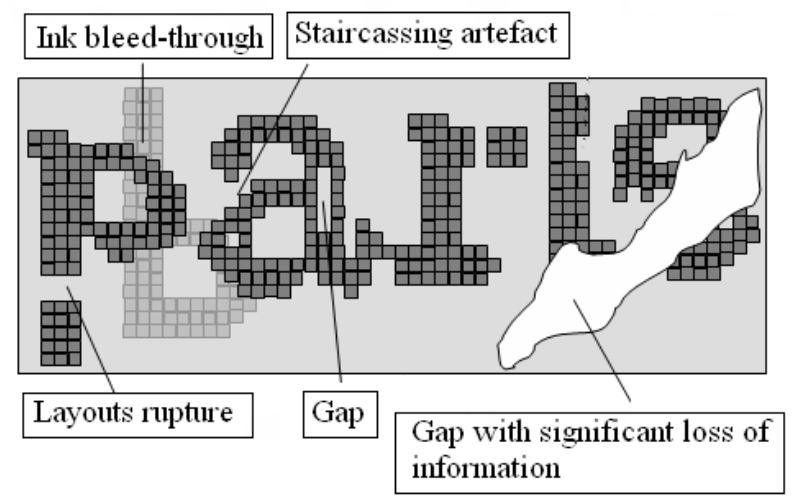

Fig. 2 Illustration of noisy foreground degradations on a synthetic image.

We can distinguish different causes of damage that threaten foreground archival support [1]: chemical effect, biological effect, "indirect digitization" (case of microfilm use as an example), processing techniques,... This work treats mainly low resolution, lossy compression and binarization artefacts generating faded, noisy and broken characters. These treatments could introduce more or less visible image artefacts resulting in ruptures of the characters' layouts and in significant modifications along their contours. For example, the binarization is an irre- 
versible process that can transform a grayscale or a color image into a black/white image according to a predefined threshold. This process could reveal random irregularities on characters layout as noise is important in high intensity variation areas (Fig.3). It could also modify the characters topology limiting the word segmentation process (Fig.4).

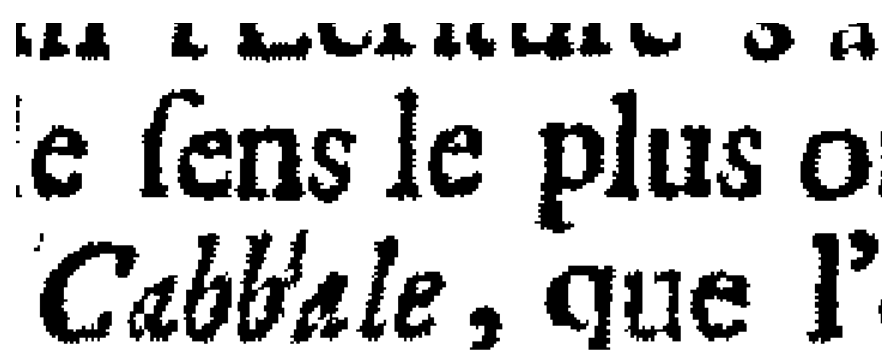

A ument images are mainly composed of features around which derivatives can be calculated precisely. Furthermore, PDE-based approaches consider images as continuous and differentiable functions. Hence, treatments through PDE-based approaches are not inherently limited by the pixel borders and can process images with very low spatial resolutions.

Fig. 3 Case of degraded characters' layouts (Trevoux).

sual quality.

The adaptation of PDE approaches in our context of restoring degraded forms of letters seems very promising. In fact, the choice of PDE is accounted for their local formulations which are well adapted to deal with local data corruption. Moreover, PDE formulation is mainly based on image derivatives that are much better defined in texrery low spatial resolutions.

\section{A study of PDE-based approaches}

\section{Muste Love That I Sculpture: Africa's bya Daniel Pedersen}

Fig. 4 Illustration of broken and damaged characters in a low resolution document image.

\subsection{Discussion}

Noisy foreground degradations, compared to the other types of degradations, face real problems that have not been resolved yet. Indeed, the existing state-of-the-art presents some contributions in this field mainly by defining a model reflecting more or less character degradations. However, no generic model describing all possible distortions has been published [33]. Moreover, virtual restoration using a particular model requires the definition of hypothesis applicable only to very specific conditions of acquisition. A simple change of conditions taken into account degrades the performance of the model. Another solution, known by its ability to model the images in a continuous space, is based on partial differential equations. This solution has been successfully evaluated for the restoration of natural images mainly in image denoising or noise removal. This treatment is necessary to facilitate further processings and even to improve the vi-
Since they are originally created to describe physical laws and natural motions of mechanical objects and fluids, PDEs have been deeply studied and extended to other branches of mathematics and physics. The PDEbased formulation enables the regularization of an image through successive iterations. In this study, we will focus on PDE-based approaches useful for noise removal and edge enhancement. We could roughly classify these different approaches into two categories (linear/nonlinear) according to the nature of the treatment. Linear PDEapproaches apply local operators uniformly to a noised image leading to isotropic smoothing. As Kœindering [41] points out, these approaches generate a smoothing process with a Gaussian kernel that erases geometrical aspects of the image :

$\left\{\begin{array}{l}I_{t}=\operatorname{div}(\nabla I) \Leftrightarrow I_{t}=I_{0} \otimes G_{\sigma=\sqrt{2 t}} \\ I_{0}=I_{\text {Noisy }}\end{array}\right.$

Hence, important features such as edges are blurred and become harder to identify.

Nonlinear algorithms overcome this problem since they tend to preserve these features during smoothing (Fig.5). The main idea underlying such algorithms is to introduce image dependent diffusivities adapting the treatment to the local properties of the image. However, according to the used diffusion coefficient, nonlinear diffusion processes could be isotropic if they are driven by a scalarvalued diffusivity or anisotropic in the case of a matrixvalued diffusion tensor. Either scalar or matrix-valued diffusivity tends to control the smoothing effect according to the local geometry of the image. 


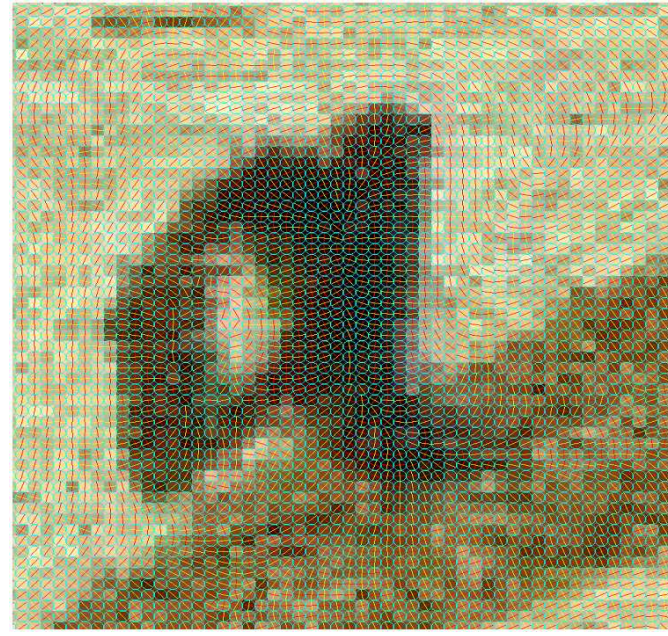

(a) Case of linear isotropic diffusion

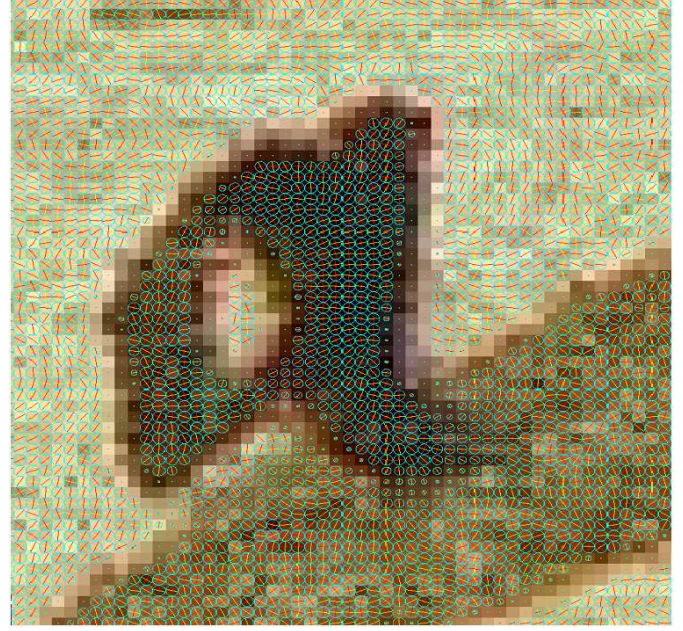

(b) Case of nonlinear isotropic diffusion

Fig. 5 Impact of linear and nonlinear isotropic diffusion on a test image.

\subsection{Nonlinear isotropic diffusion}

Perona and Malik's work $[42,43]$ on nonlinear isotropic diffusion has been the first issue in the area. In particular, a diffusion coefficient function is chosen to be inversely proportional to the image's gradient (magnitude). Therefore, the diffusion flow increases within homogeneous regions where the gradient tends to be small and decreases across non-linear discontinuities where the gradient (magnitude) tends to be small and decreases across non-linear discontinuities where the gradient (magnitude) is very high. This is clearly illustrated in the Perona-Malik equation given as:

$\left\{\begin{array}{l}I_{t}=\operatorname{div}(C(\|\nabla I\|) \times \nabla I) \\ I_{0}=\quad I_{\text {Noisy }}\end{array}\right.$

where $\|\nabla I\|$ is the gradient magnitude of the image $I$ and $C(\|\nabla I\|)$ is an edge stopping function chosen to satisfy $C(0)=1$ and $\lim _{v \rightarrow \infty} C(v)=0$.

According to this equation, we notice that the coefficient $C(\|\nabla I\|)$ serves the purpose of selecting the locations in the image for smoothing. If $C$ is chosen constant at all image locations, the diffusion equation yields isotropic Gaussian smoothing. The function $C$ could be defined by either $C(\|\nabla I\|)=\frac{1}{1+\left(\frac{\|\nabla I\|}{k}\right)^{2}}$ or $C(\|\nabla I\|)=$ $\exp \left(-\frac{\|\nabla I\|}{k}\right)^{2}$

Catté et al. [44] have proved the ill-posedness of the diffusion equation and proposed a regularized version where the cœefficient is a function of a smoothed gradient

$$
\left\{\begin{array}{l}
I_{t}=\operatorname{div}\left(C\left(\left\|G_{\sigma} \otimes \nabla I\right\|\right) \times \nabla I\right) \\
I_{0}=\quad I_{N o i s y}
\end{array}\right.
$$

Compared to the original Perona and Malik's equation, such proposition generates oriented vectors either correctly along contours or inside uniform zones (Fig.6).

In general, the nonlinear isotropic diffusion filter acts similarly to the linear diffusion filter inside uniform zones but acts differently near edges where diffusion is inhibited. Therefore, noise at edges cannot be eliminated successfully by this process. The latter was further extended to another nonlinear process which is anisotropic and driven by an adapted diffusion tensor $[45,46]$ instead of a scalar diffusivity (Fig. 7).

\subsection{Nonlinear anisotropic diffusion}

A nonlinear anisotropic diffusion equation driven by diffusion tensor is given as :

$\left\{\begin{array}{l}I_{t}=\operatorname{div}(D(J) \nabla I) \\ I_{0}=I_{\text {Noisy }}\end{array}\right.$

where $D$ is an anisotropic diffusion tensor which depends on the image via the structure tensor $J$ defined as

$J=J_{\rho}\left(\nabla I_{\sigma}\right)=G_{\rho} *\left(\nabla\left(G_{\sigma} \otimes I\right) \nabla\left(G_{\sigma} \otimes I\right)^{t}\right)$

Here $G_{\rho}$ and $G_{\sigma}$ are Gaussian convolution Kernels. $D(J)$ is performing as an edge stopping function to preserve edges.

For $D=C(\|\nabla I\|)\left(\begin{array}{ll}1 & 0 \\ 0 & 1\end{array}\right)$, we have the nonlinear isotropic diffusion equation of Perona-Malik.

The tensor $J$ could be defined in its orthonormal system of eigenvectors describing the directions where the local contrast is maximal or minimal. This contrast is measured by its eigenvalues. It is defined as :

$J=\lambda_{-} \times \Theta_{-} \Theta_{-}^{t}+\lambda_{+} \times \Theta_{+} \Theta_{+}^{t}$ 


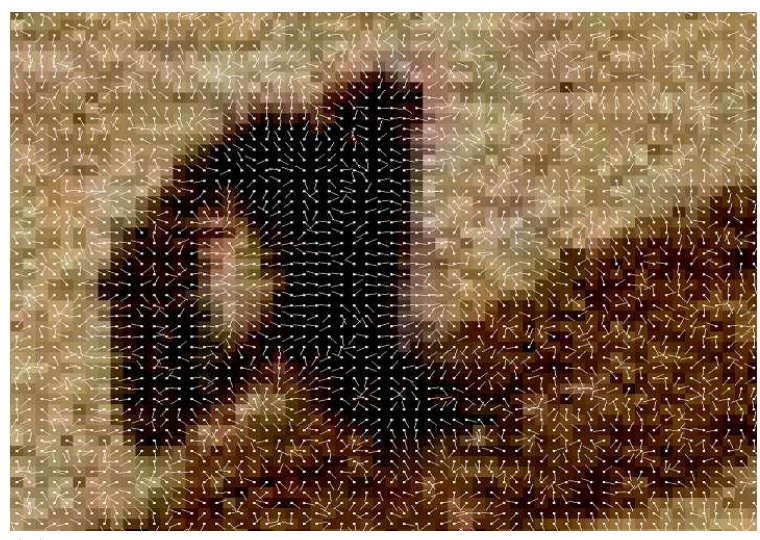

(a) Orientation of the gradient vectors in the case of the Perona-Malik process

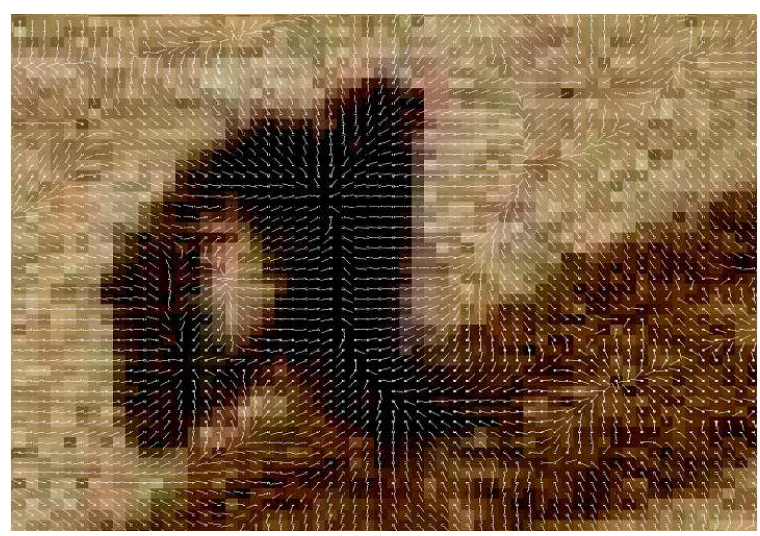

(b) Orientation of the regularized gradient vectors in the case of the Catté et al. process

Fig. 6 Illustration of the difference between the Perona-Malik diffusion equation and its regularized version proposed by Catté et al.

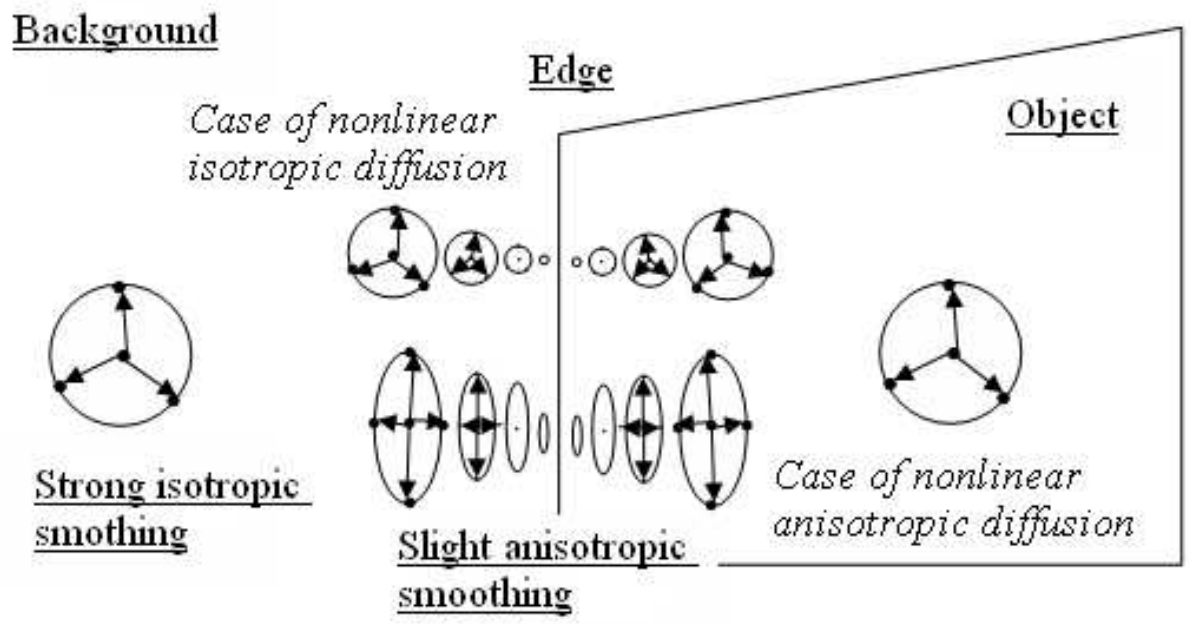

Fig. 7 Nonlinear isotropic and anisotropic diffusion processes are different near contours but similar inside uniform zones.

where $\lambda_{+/-}$and $\Theta_{+/-}$are the eigenvalues and the eigenvectors of the tensor field $J$.

Therefore, the diffusion tensor is given by:

$D=f_{+}\left(\lambda_{+}, \lambda_{-}\right) \times \Theta_{-} \Theta_{-}^{t}+f_{-}\left(\lambda_{+}, \lambda_{-}\right) \times \Theta_{+} \Theta_{+}^{t}$

$f_{+}\left(\lambda_{+}, \lambda_{-}\right)$and $f_{-}\left(\lambda_{+}, \lambda_{-}\right)$are two functions satisfying the two local constraints:

1. Edge preservation: if $\lambda_{+/-} \gg 0$, the smoothing process should be anisotropic and directed along the edge direction $\Theta_{-}$.

2. Smoothing within homogenous areas: if $\lambda_{+/-} \approx 0$, the smoothing process should be isotropic.

Among the promising anisotropic PDE-based approaches, we have noticed the Weickert approach $[47,46]$ and the R. Kimmel et al. approach known as the beltramiflow $[48,49]$. The concept is the same but the values of $f_{+/-}\left(\lambda_{+}, \lambda_{-}\right)$are different (Tab.1). Both of these approaches are based on gradient divergence. D. Tschumperlé [50] introduced a new formulation based on the computation of the trace operators and the Hessian matrix instead of the divergence. Such formulation is given by

$\left\{\begin{array}{l}I_{t}=\operatorname{trace}(D H) \\ I_{0}=I_{\text {Noisy }}\end{array}\right.$

\subsection{Discussion}

This section presents some typical PDE-based approaches commonly known for their performance in noise removal while preserving important features. The behaviour of each approach in treating local image properties changes according to the function $f_{+/-}$(Tab.1). To illustrate this idea, we give in figure 8, the diffusivity matrix $D$ for the different studied functions $f_{+/-}$. Each matrix, drawn in the case of the image of a letter "a", is characterized by 


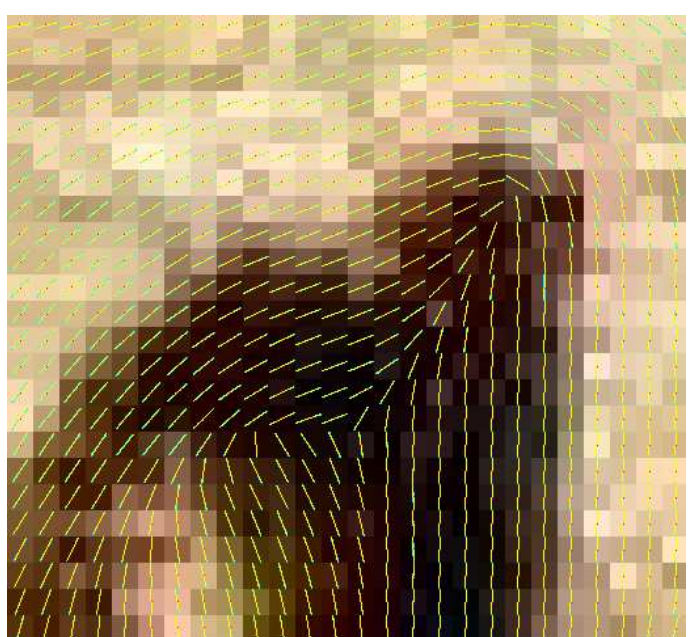

(a) Case of the Weickert diffusivity process

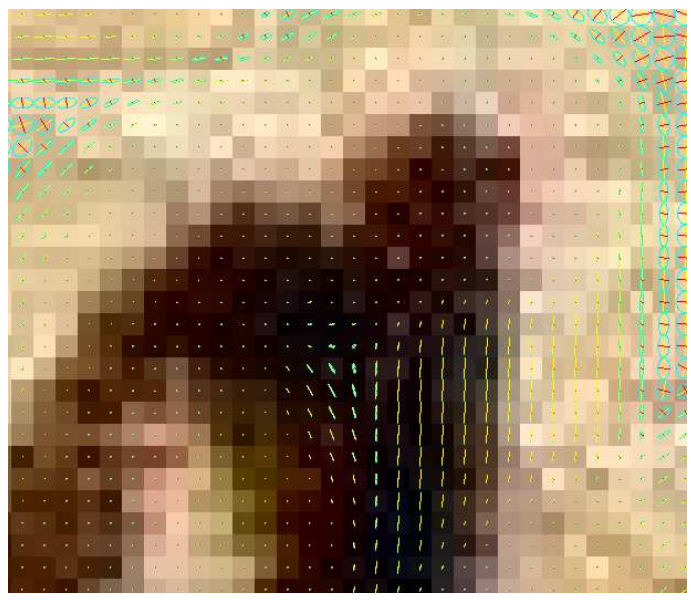

(c) Case of the Beltrami diffusivity process

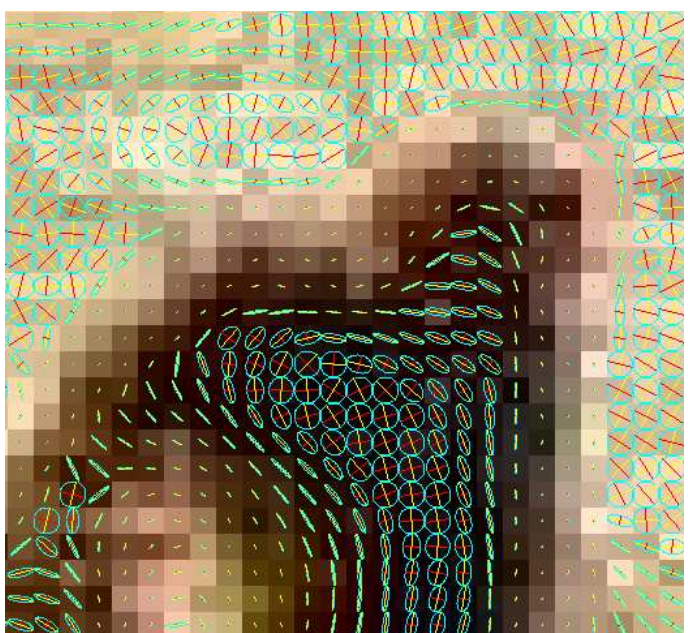

(b) Case of the Tschumperlé diffusivity process

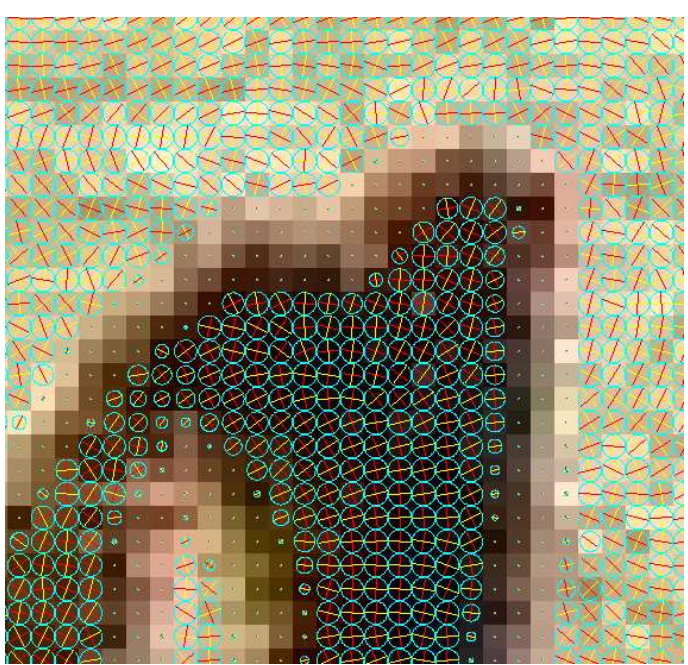

(d) Case of the Perona-Malik-Catté diffusivity process

Fig. 8 The behaviour statment of different nonlinear anisotropic diffusion approaches around contours and corners.

\begin{tabular}{|c|c|c|}
\hline Perona-Malik & $f_{+}\left(\lambda_{+}, \lambda_{-}\right)=f_{-}\left(\lambda_{+}, \lambda_{-}\right)=\exp -\left(\frac{\left\|G_{\sigma} \otimes \nabla I\right\|}{K}\right)^{2}$ & $I_{t}=\operatorname{div}\left(C(\|\nabla I\|)\left(\begin{array}{ll}1 & 0 \\
0 & 1\end{array}\right) \nabla I\right)$ \\
\hline Weickert & $\begin{array}{rlrl}f_{+}\left(\lambda_{+}, \lambda_{-}\right) & =\alpha+(1-\alpha) \exp \left(\frac{-C}{\left(\lambda_{+}-\lambda_{-}\right)^{2}}\right) & \text { if } \lambda_{+} \neq \lambda_{-} \\
& = & \text {else } \\
f_{-} & = & \alpha=0.001 & \end{array}$ & $I_{t}=\operatorname{div}(D \nabla I)$ \\
\hline Beltrami & $\left\{\begin{array}{l}f_{+}\left(\lambda_{+}, \lambda_{-}\right)=\sqrt{\frac{1+\lambda_{+}}{1+\lambda_{-}}} \\
f_{-}\left(\lambda_{+}, \lambda_{-}\right)=\sqrt{\frac{1+\lambda_{-}}{1+\lambda_{+}}}\end{array} I_{t}=\frac{1}{\sqrt{\left(1+\lambda_{-}\right)\left(1+\lambda_{+}\right)}} \operatorname{div}(D \nabla I)\right.$ & $I_{t}=\operatorname{div}(D \nabla I)$ \\
\hline Tschumperlé & $\left\{\begin{array}{l}f_{+}\left(\lambda_{+}, \lambda_{-}\right)=\frac{1}{\sqrt{1+\lambda_{+}+\lambda_{-}}} \\
f_{-}\left(\lambda_{+}, \lambda_{-}\right)=\frac{1}{1+\lambda_{+}+\lambda_{-}}\end{array}\right.$ & $I_{t}=\operatorname{trace}(D H)$ \\
\hline
\end{tabular}

Table 1 Different functions $f_{+/-}$corresponding to different anisotropic diffusion approaches.

the shape of an ellipse directed by $\Theta_{+}$and its axis length is equal to $\sqrt{f_{+/-}\left(\lambda_{+}, \lambda_{-}\right)}$(Fig. 9). 


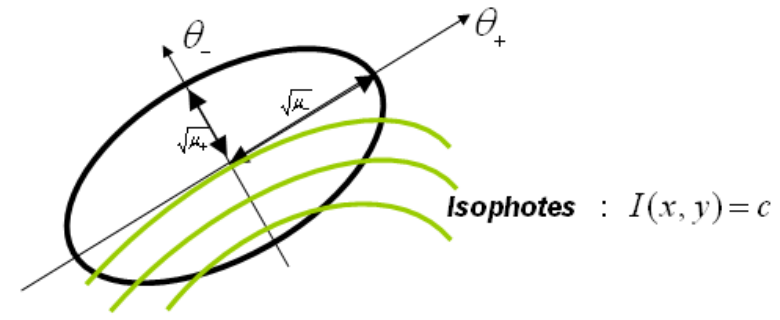

Fig. 9 The tensor's structure in the case of nonlinear anisotropic diffusion where $f_{+}\left(\lambda_{+}, \lambda_{-}\right)=\mu_{+}$and $f_{-}\left(\lambda_{+}, \lambda_{-}\right)=\mu_{-}$.

With the illustration given in figure 9 , we are able to highlight the advantages and the disadvantages of the studied approaches. In fact, the diffusion filter of PeronaMalik-Catté gives very good results for the preservation of character topologies. For instance, the value of the local smoothed gradient, which weights the diffusion, avoids corner rounding and preserves edges. Nevertheless, the Perona-Malik-Catté filter does not smooth contours disturbed by noise and does not ensure their continuity. The filters of Beltrami and Tschumperlé give better results as they have the property of reducing noise, smoothing contours and preserving the structures. However, they reinforce the continuity of edges very moderately. This problem was successfully studied by Weickert who proposes a new filter able to restore interrupted structures. This mentioned property is very interesting. Nevertheless, the lack of a correct treatment of the image contours curvatures remains the main drawback of this regularization process.

Table 2 summarizes the advantages and the disadvantages of Perona-Malik-Catté, Weickert, Beltrami and Tschumperlé diffusion models respectively. This table presents the behaviour of each approach in the case of noise removal, coherence-enhancing and singularity preservation as explained above.

\section{Proposition of a new PDE-diffusion approach}

\subsection{Towards a model preserving singularities}

Faced with a wide range of existing constrained and unconstrained PDE formulations, each regularization method has its advantages and its disadvantages as it was explained above. If most of them have in common noise removal with contour prservation, their ability in coherenceenhancing and corner preservation differs from one method to another. As a requirement, defining a typical regularization method combining the best of existing ones could be an interesting aim. For instance, it would be desirable to design a filter useful for noise removal with coherenceenhancing property and both corner and edges preservation. Having all these properties grouped in one filter would be very interesting mainly in the treatment of textual document images with poor quality. In fact, noise removal would be beneficial if the legibility of the text as well as the visual quality of an image are enhanced. Such enhancement is correctly achieved if this noise removal takes into account the reinforcement of broken characters (coherence-enhancing property) and adapts smoothing as much as possible to the geometry of the image. By image geometry, we mean every important feature in the image such as singularities (areas with high curvatures or right angles (Fig.10)) and edges.

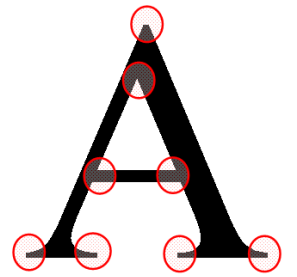

(a)

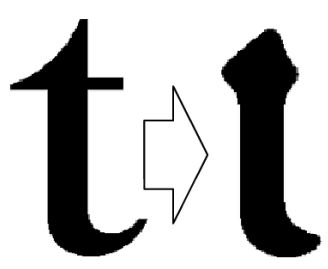

(b)
Fig. 10 (a) Localisation of singularities in the case of the letter "A", (b) Importance of the singularity details for the recognition.

For instance, singularities must be treated carefully as any modification, in the case of textual document images, could change one letter to another and consequently change the meaning. Thus, we will be faced with a meaningless textual document. For example, the loss of singularities could transform the letter " $t$ " to the letter "l" which are completely different (Fig. 10).

The Weickert coherence enhancing diffusion process, compared to the other processes, is characterized by its abilities to repair stroke discontinuities on document images. This property is a very desirable one mainly in treating broken characters of degraded textual documents. However, the Weickert process destroys singularities and leads generally to curved structures. This problem could be avoided with a direct application of the scalar diffusion introduced by Perona-Malik-Catté. This process has the interesting property to vanish when the value of the local smoothed gradient is important. Looking for an approach that avoids corner rounding and takes into account the coherence-enhancing diffusion, we propose combined Perona-Malik and Weickert processes as one approach (Fig.11).

\subsection{Description of our proposition}

Our proposition takes benefit from the scalar diffusion model of Perona-Malik and the tensor driven diffusion process of Weickert. As we have previously noticed, the filter of Perona-Malik is a special case of the Weickert 


\begin{tabular}{|c|c|c|c|}
\hline Nonlinear diffusion & edge-enhancing & coherence-enhancing & corner preservation \\
\hline \hline Perona-Malik-Catté & No & No & Yes \\
\hline Weickert & Yes & Yes & No \\
\hline Tschumperlé & Yes & No & Yes/No \\
\hline Beltrami & Yes & Yes/No & Yes \\
\hline
\end{tabular}

Table 2 A performance comparison of different nonlinear diffusion approaches.

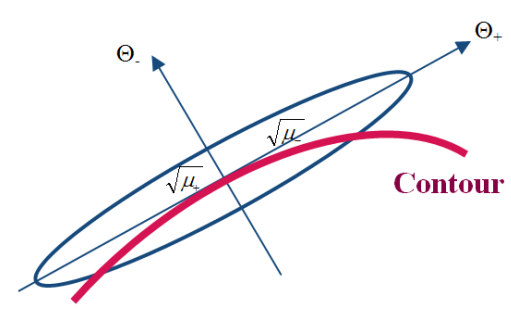

(a) Diffusivity Matrix $D^{*}$ in the case of edge processing

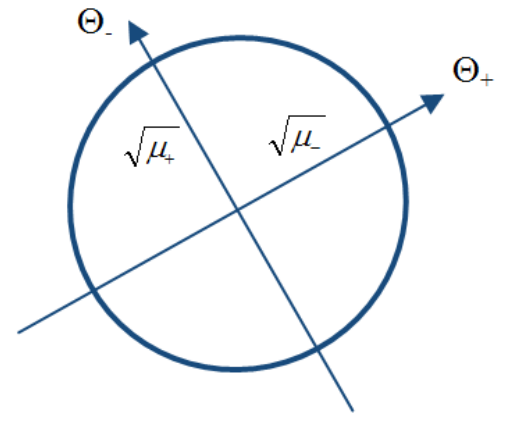

(b) Diffusivity Matrix $D^{*}$ in the case of processing areas with slight variations

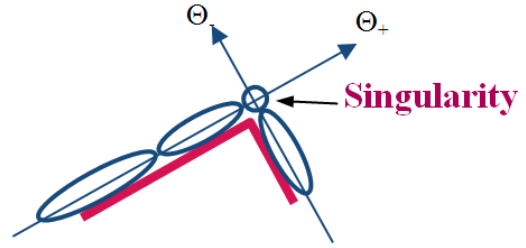

(c) Diffusivity Matrix $D^{*}$ in the case of singularity processing

Fig. 12 The shape of diffusivity matrix associated to our proposed PDE-based filter in the case of three different processed areas.

filter. Thus, the main difference between these two approaches relies on the diffusivity coefficient $\mu_{+/-}$. This coefficient in the case of the Weickert filter helps in smoothing along the flow direction. The shape of the diffusivity matrix is thus strongly anisotropic that fails to respect singularities. For the Perona-Malik filter, the diffusivity matrix has no specific orientation. It is represented by a circle that the rayon vanishes near important features (edges and corners). To take advantage of both of the previously compared filters, we propose to maintain in the new filter the Weickert diffusion oriented in the direction of $\Theta_{+}$but to change the diffusivity coefficient $\mu_{+/-}$. This new cofficient is required to ensure a correct trade-off between complete noise removal and important feature preservation. To this end, we differentiate between diffusion near a contour where the values of $\lambda_{+}$ and $\lambda_{-}$are large and the diffusion near a corner where $\lambda_{+}$or $\lambda_{-}$presents high values. The proposed solution to such suggestion is to define the values of $\lambda_{+}$and $\lambda_{-}$ separately in the diffusivity coefficient $\mu_{+/-}$. After several attempts, we succeed in designing a stable filter that satisfies all the imposed requirements: Our new filter employs two thresholds $K_{+}$and $K_{-}$useful for identifying the diffusion along the respective $\Theta_{+}$and $\Theta_{-}$axies. For instance, the quantity of diffusion changes with the encountered feature: contour or singularity. Therefore, the parameters $K_{+}$and $K_{-}$are used to define the sensitivity threshold necessary to detect a variation in the image.

In its complete form, the equation of the proposed diffusion filter is written as follows:

$$
\begin{aligned}
& \left\{\begin{array}{l}
I_{t}=\operatorname{div}\left(D^{*} \times \nabla I\right) \\
I_{0}=I_{\text {Noisy }}
\end{array}\right. \\
& \left\{\begin{array}{c}
D^{*}=\exp \left(-\frac{\lambda_{-}}{K_{-}}\right) \times \Theta_{+} \Theta_{+}^{t}+\exp \left(-\frac{\lambda_{+}}{K_{+}}\right) \times \Theta_{-} \Theta_{-}^{t} \\
D^{*}=\frac{1}{1+\left(\frac{\lambda_{-}}{K_{-}}\right)} \times \Theta_{+} \Theta_{+}^{t}+\frac{1}{1+\left(\frac{\lambda_{+}}{K_{+}}\right)} \times \Theta_{-} \Theta_{-}^{t} \\
\Theta_{+/-}, \quad \lambda_{+/-} \text {eigenvectors and eigenvalues of } T_{\sigma}^{\rho}
\end{array}\right.
\end{aligned}
$$

As this PDE is designed to smooth edges, reduce noise in uniform zones and preserve singularities, we define a specific treatment for each of these situations as follows (Fig.12):

- Near an edge, we have $\lambda_{+}>>\lambda_{-}, \lambda_{+}>K_{+}$and $\lambda_{-}<K_{-}$. In this case, the diffusivity cœfficient $\mu_{-}$ (respectively $\mu_{+}$) is close to 0 (respectively to 1 ) in the direction of $\theta_{-}$(respectively $\left.\theta_{+}\right)$. A treatment as such is similar to that of Weickert. It enhances the consistency of features and accentuates smoothing along edges (Fig.12(a)).

- In uniform areas with low intensity variation, we have $\lambda_{+}<K_{+}$and $\lambda_{-}<K_{-}$. In this case, the diffusivity matrix $D^{*}$ becomes totally isotropic as both of the diffusivity cofficients $\mu_{+}$and $\mu_{-}$are close to 1 . The diffusion here is equivalent to that done with the heat equation erasing all the small variations (Fig.12(b)).

- Near a singularity, we have $\lambda_{+}>K_{+}$and $\lambda_{-}>K_{-}$. In this case, the diffusivity cofficients $\mu_{+}$and $\mu_{-}$ are close to 0 . The diffusion process, simulated here for the Perona-Malik process, is stopped in order to preserve right angles and corners (Fig.12(c)). 

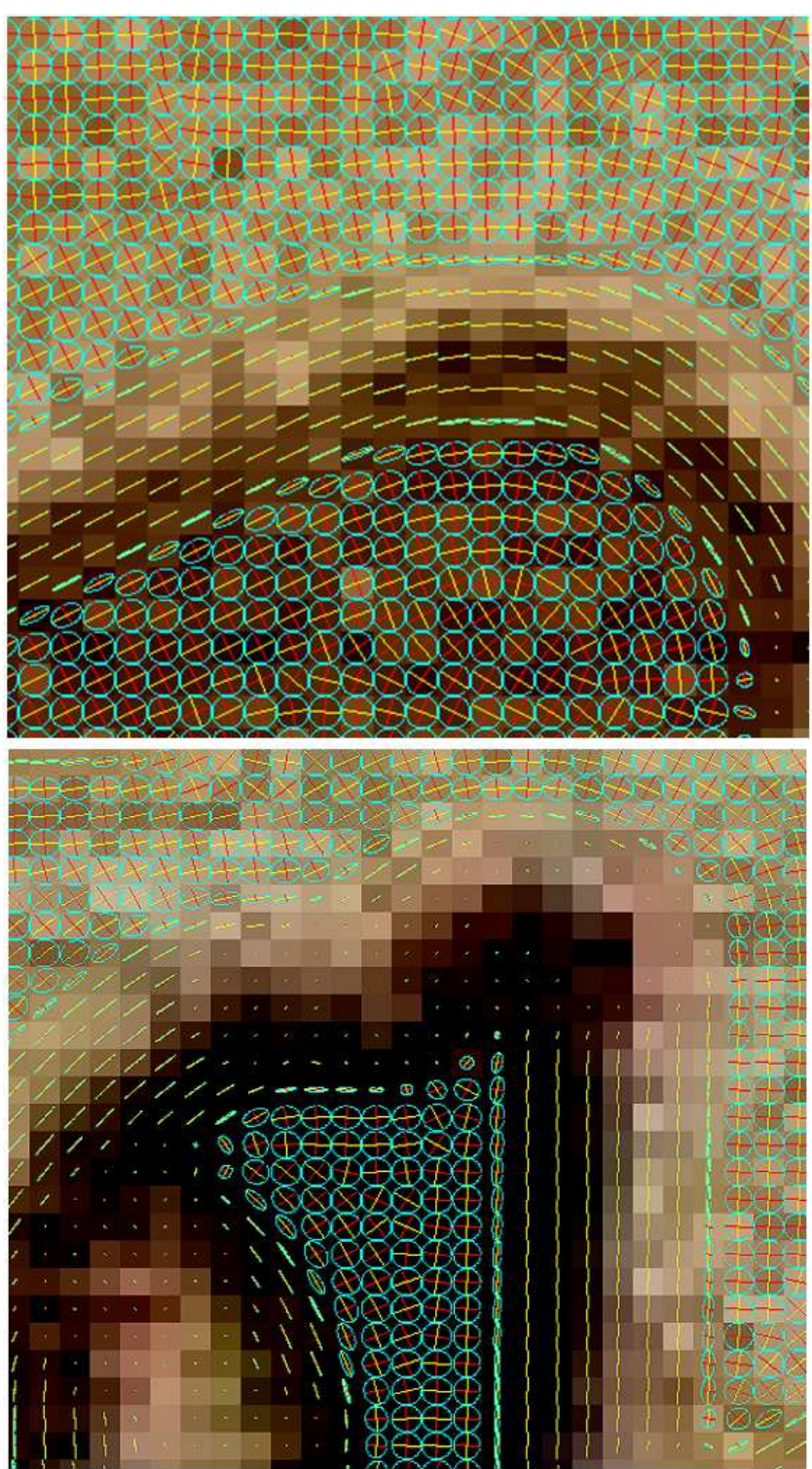

Fig. 11 The behavior statement of our proposition around contours and corners (a mixture of both of Perona-Malik and Weickert behavior processes(Fig.8)).

\subsection{Estimation of the parameters}

To evaluate the performance of the proposed filter, in this section we will study the impact of different range values of $K_{+}$and $K_{-}$. Figure 13 illustrates, as an example, the treated images with different parameter values of $K_{+}$and $K_{-}$. We clearly notice a visual quality improvement of the noisy original image. Hence, noise is eliminated in the case of the different restored images. The parameters $K_{+}$and $K_{-}$affect mainly slight parts; in the case of equal values (Fig.13(b)), they tend to erase these parts, otherwise, their extremities are prolonged in their flow direction (Fig.13(c)) and even the reglure could appear (Fig.13(d)).

$K_{+}$is the sensitivity threshold useful for the preservation of the edges. When the value of $K_{+}$increases, edges of low contrast objects in the image could be erased. This is due to the fact that, in this case, the value of $\lambda_{+}$is high near a contour whatever the value of $\lambda_{-}$is. As an example, we noticed in figure $13(\mathrm{~d})$ that the termination of the letter "a" disappears when the contours variation are lower than $K_{+}$. Besides the threshold $K_{+}$, our proposed PDE-based filter introduces another threshold value, $K_{-}$, useful for the preservation of singularities. When the value of $K_{-}$increases, singularities in the image could be erased. In fact, the value of $\lambda_{-}$increases near a singularity as it represents a complex structure compared to a simple line or a uniform area.

By comparing both of the values of $K_{-}$and $K_{+}$, we can notice two situations:

- If $K_{-} \gg K_{+}$, then the proposed filter processes orient structures by smoothing along the flow direction. This represents the basic treatment of the Weickert coherence-enhancing diffusion.

- If $K_{+} \gg K_{-}$, then we have a better preservation of image singularities and a significant loss of contours with slight variations. However, with an important value of $K_{+}$, the image contours will no longer be preserved.

Moreover, as we clearly notice from the previous section, the estimation of the parameters $K_{+}$and $K_{-}$for a given image depends greatly upon the eigen-values $\lambda_{+}, \lambda_{-}$. These values vary according to the smoothing parameters $\sigma$ and $\rho$ of the tensor field $T_{\sigma}^{\rho}$. If the regularization parameter $\sigma$ is generally equal to 0.5 , the parameter $\rho$ depends upon the image contents and controls the correct estimation of the anisotropic diffusion orientation. For document images, $\rho$ varies according to the noise, the continuity of strokes and the text heights. In fact, poorly degraded documents characterized by a reduced noise along characters' contours and continuous strokes and even document images with small text heights require small $\rho$ values varying from 0.5 to 1.5 . For strongly degraded documents having large discontinuities or for documents having high text heights, $\rho$ must be fixed between 1.5 and 3 . In general, the values $\sigma=0.5$ and $\rho=1.5$ work pretty well for a great majority of document images.

The eigen-values are naturally square values since they are calculated from the tensor-matrix having also square values. This explains the work of the PeronaMalik [42]. For instance, the exponential argument is not put in square but contains the eigen-value divided directly by the parameter $K$. This parameter stops the diffusion for real contours and boosts the diffusion in noisy regions. In our case, the eigen-values of the tensor matrix replace the gradient magnitude argument in the 


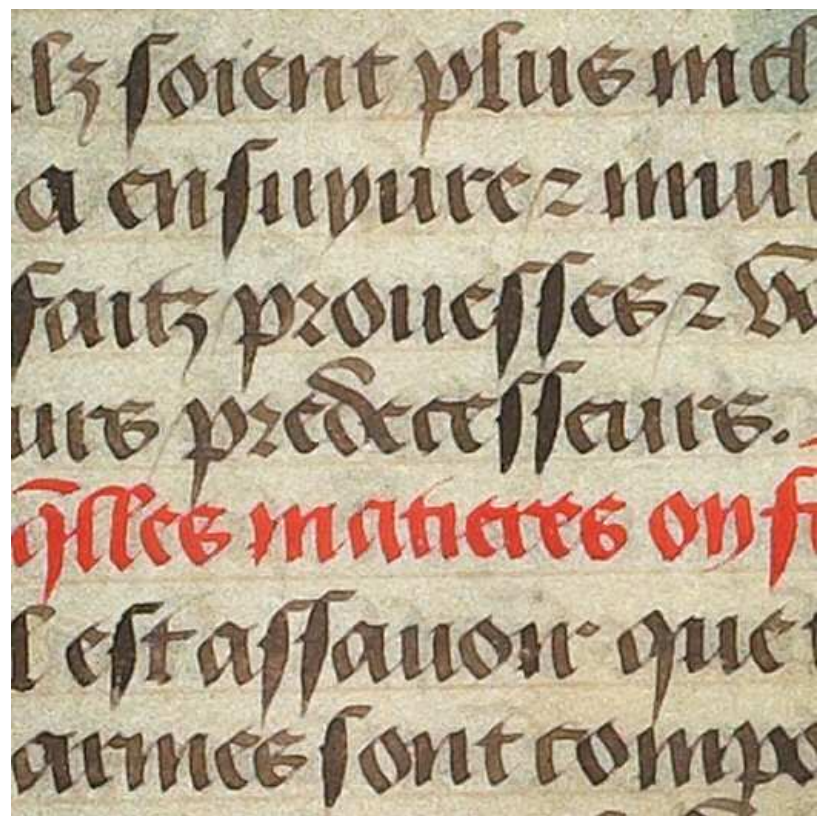

(a)

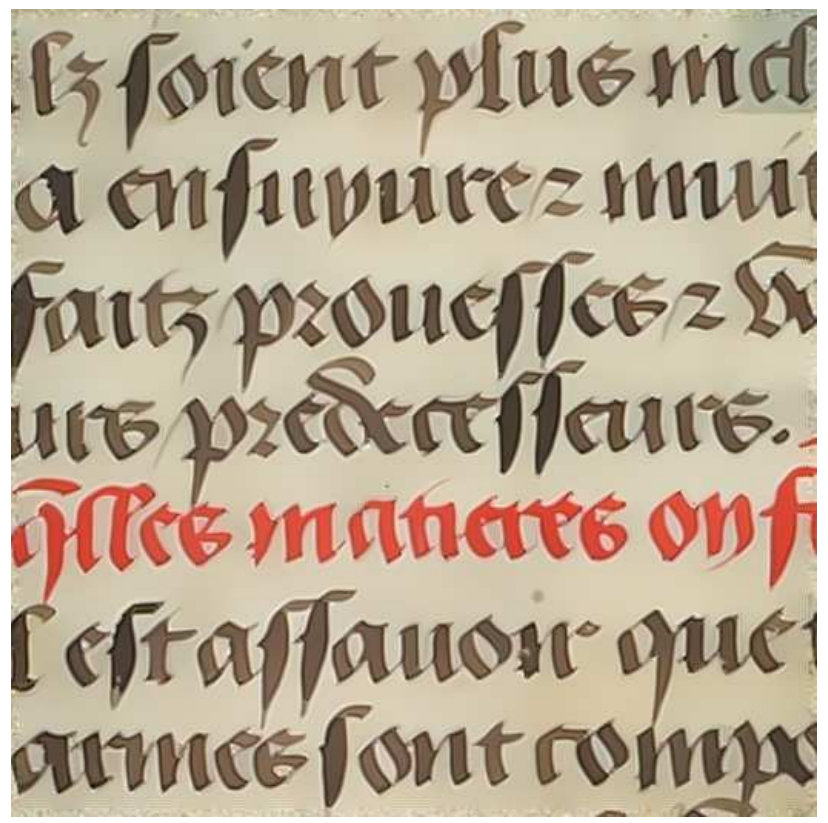

(c)

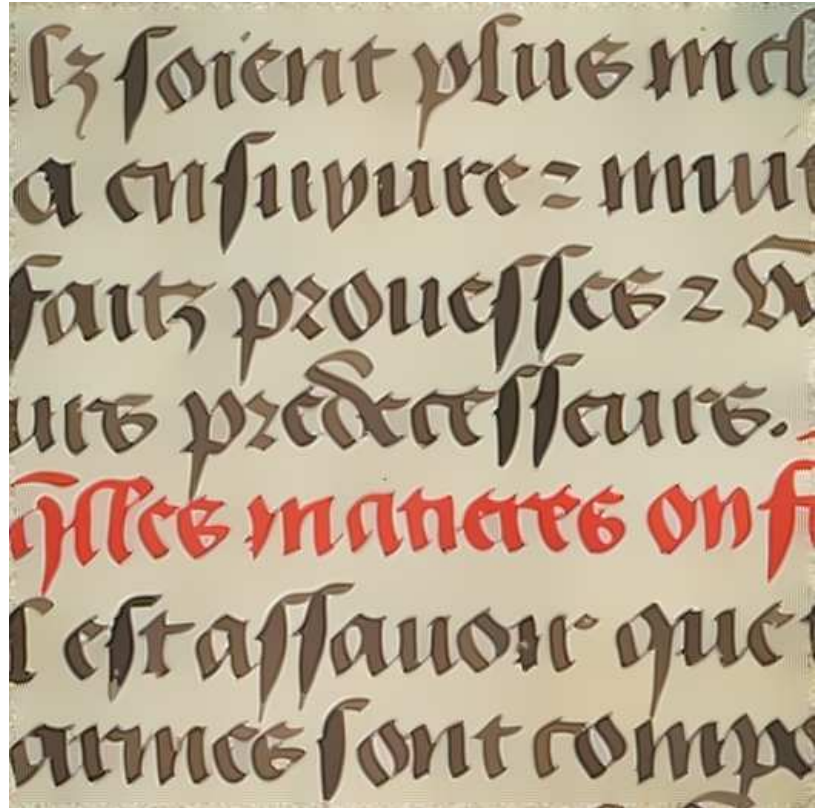

(b)

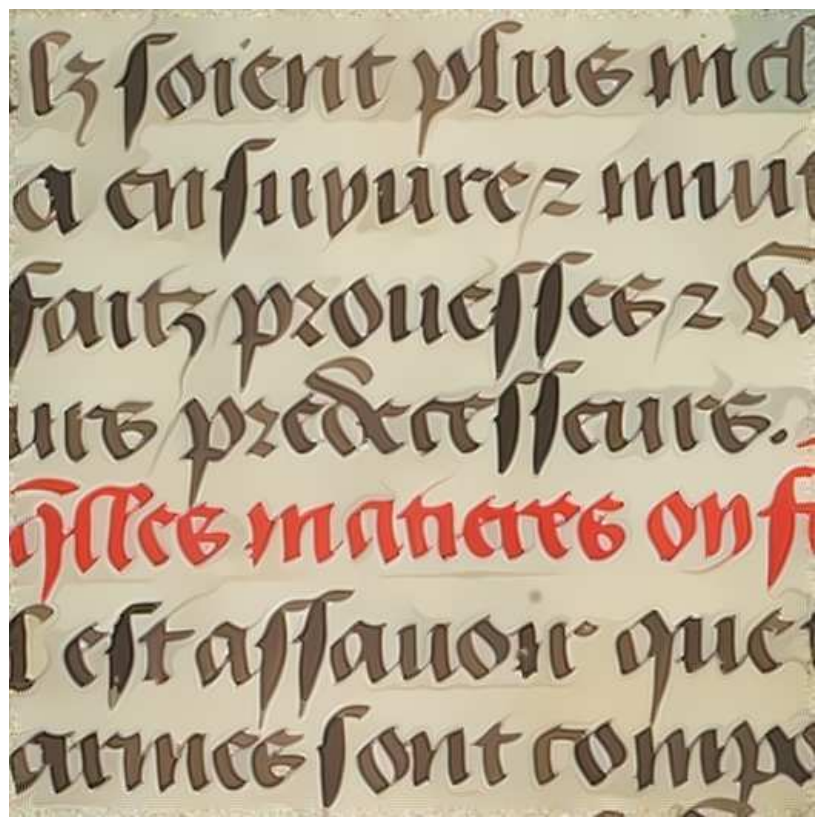

(d)

Fig. 13 (a) The original image and the restored image with $f_{1}, \sigma=0.5, \rho=1.5$, (b) $K_{+}=K_{-}=128$ (c) $K_{+}=60$, $K_{-}=128,(\mathrm{~d}) K_{+}=20, K_{-}=128$.

control of the diffusion process. We must then automatically find the parameter $K$ useful for both contours and singularity preservation.

Figure 14 shows the 2D-joint histograms measuring eigen-values for a large range of $\lambda$ eigen-values (Fig 14 b) the statistical co-occurrences of eigen-value variation, mainlyAnother example given with the classical image of Lena the values of $\lambda_{+}$and $\lambda_{-}$calculated simultaneously in is characterized by a limited number of singularities and each pixels. This histogram definition differs between natural and document images. For example, the image of Barbara shows a great number of contours and straight lines but a few number of singularities like corners. For this reason, the statistical cooccurrence matrix of this image shows a high number of pixels having high $\lambda_{+}$ eigen-values for a large range of $\lambda_{-}$eigen-values (Fig.14.b)
Another example given with the classical image of Lena 


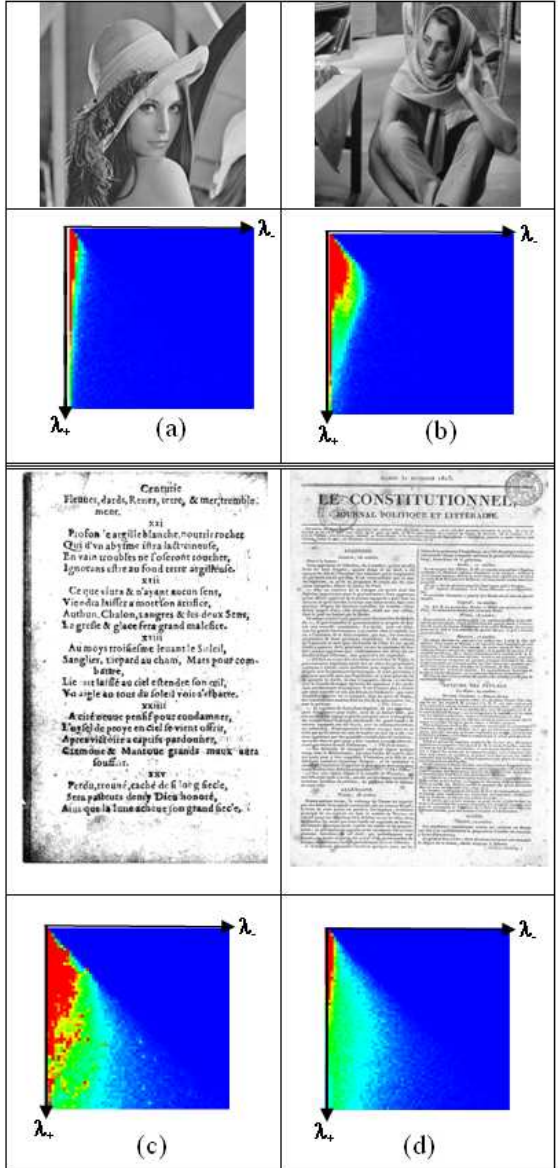

Fig. 14 2D-joint histograms representing the statistical cooccurrence of eigen-values $\lambda_{+}$and $\lambda_{-}$for natural images of (a) Lena and (b) Barbara and for document images of (c) the book of Nostradamus (pp.18/127)[54] and (d) a Journal in low resolution [55].

very few number of contours. The associated $2 \mathrm{D}$-joint histogram contains a great number of small $\lambda_{-}$eigenvalues for a large range of $\lambda_{+}$eigen-values (Fig.14.a). Both of the examples cited above give results completely different from those obtained on document images. The 2D-joint histograms of these images show the occurrence of large eigenvalues $\lambda_{+}$and $\lambda_{-}$, proof of the presence of numerous contours and singularities (Fig.14.c, Fig.14.d).

Since $\lambda_{+}$reaches high values along contours, it cannot be used for an estimation of the $K$ parameters. Nevertheless, the $\lambda_{-}$eigen-value which reaches high values around singularities, could be used for the estimation of the parameters $K$ in the case of document images processing. In order to preserve these singularities, we fix the parameter $K_{-}$by taking $10 \%$ of the $\lambda_{-}$maximum values measured in the entire image. We assume that for document images written in Latin script, on average, $10 \%$ of the contours are located around singularities. Hence, this estimation allows to preserve main singularities like corners and strokes endings. For the parameter $K_{+}$, we propose to fix it at half the value of $K_{-}$in order to reinforce efficiently strokes continuities.

$$
\left\{\begin{array}{l}
K_{-}=0.1 \times \max \left(\lambda_{-}\right) \\
K_{+}=\quad \frac{K_{-}}{2}
\end{array}\right.
$$

\section{Authun Aothun}

(a)

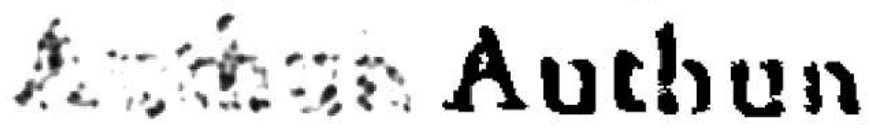

(c)

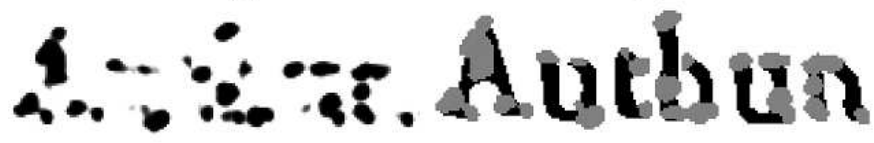

(e) (f)
Fig. 15 The effect of an automatic threshold $K_{+}$and $K_{-}$ definition for both contours detection and singularities preservation with $\sigma=0.5$ and $\rho=1.5$. From left to right and top to down respectively: (a) Original image of a word [54], (b) Values of $\exp \left(\lambda_{+} / K_{+}\right)$representing the contours to smooth on the image a), (c) Values of $\exp \left(\lambda_{-} / K_{-}\right)$which localize the singularities on a) before restoration, (d) Restored image, (e) Values of $\exp \left(\lambda_{-} / K_{-}\right)$which localize the singularities on d), (f) Restored image superposed with singularities displayed in gray.

Figure 15 details the automatic estimation of the $K$ parameters. For instance, the figure 15.a shows the original image to restore followed by the figure 15.b representing the diffusion coefficients $\exp \left(\lambda_{+} / K_{+}\right)$defined with the estimated $K_{+}$parameter. These coefficients help to localize the contours to smooth with an anisotropic diffusion. Another diffusion coefficients defined with the automatic threshold $K_{-}$are calculated as $\exp \left(\lambda_{-} / K_{-}\right)$. This equation extracts from the processed image all the singularities necessary to preserve. Figure 15.c and figure 15.e give the extracted singularities respectively before and after the restoration process. The result of the superposition between different singularities and the restored image is shown in the figure 15.f. It highlights the difficulties to make a difference between real singularities like strokes endings, true intersections and false singularities like noisy discontinuities and small holes. This problem is not really new, Perona and Malik have already pointed out the difficulties to fix the threshold $K$ in order to make the difference between contours and noise. Our automatic parameterization works for most document images. But the automatic parameter may be tuned to special fonts like Gothic or Fraktur typeface which show too many angles and singularities. It is also the same for other scripts like Arabic, Hebrew, Chinese... 


\subsection{Limits of the coherence enhancing diffusion}

The tensor driven diffusion process, proposed by Weickert, leads to good results in case of anisotropic structure. In theory, the Weickert model reinforces continuities of strokes (Fig.16.a). But in practice, we have observed that this restoration is not achieved when the flows cannot be aligned in five cases:

- The smoothing parameter is not correctly fixed for a right estimation of the directions of the flow (Fig.16.b).

- The junctions are not aligned (Fig.16.c).

- The endings around discontinuities are detected as singularities (Figure 16.d)

- The endings appear locally with different orientations (Fig.16.e)

- The discontinuities make a "T" junction (Fig.16.f)

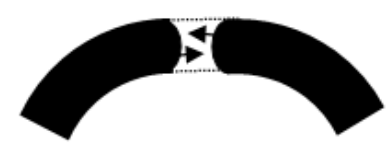

(a)

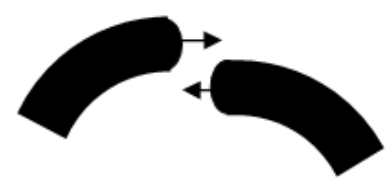

(c)

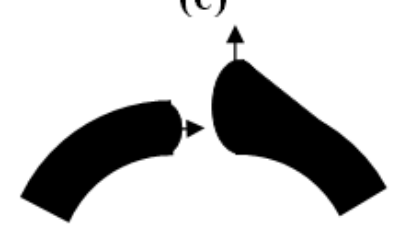

(e)

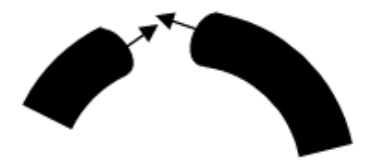

(b)

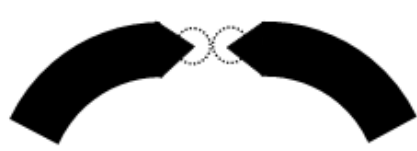

(d)

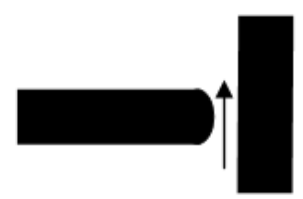

(f)
Fig. 16 impact of the flow direction, represented by a black arrow, in the reconstruction process. From Left to right and from top to down respectively: (a) correct alignement, (b) $\rho$ is not correctly fixed, (c) Junction not aligned, (d) endings detected as singularities, (e) local endings orientations, (f) "T" junctions.

Figure 17 gives an example of the coherence enhancing diffusion effects for dot matrix printings. With an optimal smoothing parameter $\rho$, we still observe errors of stroke reconstruction due to the wrong alignment of the flow. In some cases (for example the characters "à", " $\mathrm{h}$ " and "e" in the last line), the restoration can modify the topology of some characters because of the disorientation of the flow. But in most cases, the restoration repairs the shapes of characters.

\section{Autreme Autreme d'essenc d'essenc aे lheure ò l'heure}

Fig. 17 Restoration of dot matrix printings by the proposed filter with automatic parameters; respectively from left to right the original image and its restored version.

\section{Experimental results : Application for image denoising}

\section{1 document image quality measures}

Quality measures necessary to compare the visual difference between two images are a good issue in ranking, evaluating and optimizing image restoration algorithms. Two solutions are possible to measure as such difference; subjective and objective measure. For the subjective measure, it is a good solution since a human is the ultimate viewer but it is very costly. The objective measure is easier to implement and to test but it does not always agree with the subjective one.

In this section, we choose using objective measure by testing the most popular distortion measures, such as the Peak Signal-to-Noise-Ratio (PSNR), the Improvement in Signal-to-Noise Ratio (ISNR) and the Mean Square Error (MSE), used in image and video processing. This choice is made in order to make the results generated on document images comparable to the literature.

For an image processing system with as the input image $I(x, y)$, as the processed output image $\widehat{I}(x, y)$ and as the dimensions of the images $(\mathrm{w}, \mathrm{h})$, the MSE is defined as

$$
M S E(d B)=\frac{1}{w \times h} \sum_{y=0}^{h-1} \sum_{x=0}^{w-1}[I(x, y)-\hat{I}(x, y)]^{2}
$$

The MSE measures the average of the square of the errors between two images. The growth of the assumed amount of measurement error, caused by the presence of several different features between the two images, leads to larger MSE values.

PSNR is defined as the ratio of peak signal power to average noise power.

$P S N R(d B)=20 \log \left(\frac{255}{\sqrt{M S E}}\right)$

In order to assess the quality and measure the improvement, we compare the PSNR of the degraded image with the PSNR of the restored image. An efficient image restoration process must amplify the PSNR. For example, the reduction of the noise by a factor of 2 increases 
the value of PSNR to $6 \mathrm{~dB}$. From the mathematical formulae given above, we notice the inverse relation between the MSE and PSNR. Thus, lesser errors lead to a lower value for MSE and a high value of PSNR.

Instead of making the difference between the PSNR before and after restoration, we can use directly the Improvement in Signal-to-Noise Ratio (ISNR) measure.

$$
I S N R(d B)=10 \log \left(\frac{\|G-I\|^{2}}{\|\hat{I}-I\|^{2}}\right)
$$

where $I, G$ and $\hat{I}$ are the original, degraded and restored images respectively. The larger is the Improvement in Signal to Ratio Noise value, the better is the restoration.

Certainly, these classical scalar-valued image quality measures are very commonly used in video and image processing; but they remains not often appropriate to document image processing since they are a point-based measurement and mutual relations between pixels are not taken into account. For instance, a simple document image with a strong additive noise is shown in figure 18. Although the document is characterized by a low PSNR, it remains readable. Another extract of textual image given in figure 4 has little degradation especially located around significant features. For this case, the PSNR value is high but the text remains human and machine (OCR) unreadable.

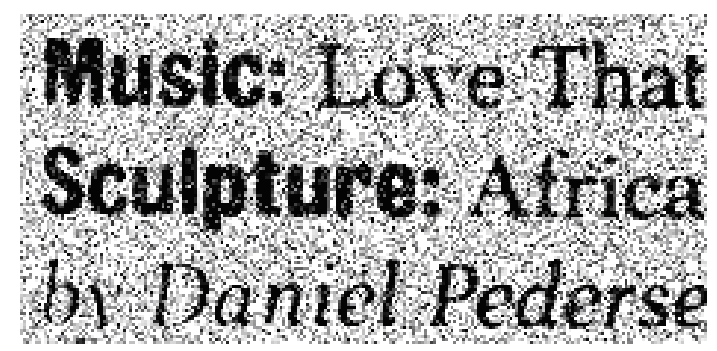

Fig. 18 An extract with strong additive noise and low PSNR.

We must notice that document images are generally characterized by the presence of a great amount of pixels without no specific/important information (the paper background) that influences statistically the MSE measure or the correlation measure. Consequently, degraded images which are no more readable keep almost the same MSE, correlation rate and PSNR compared to the same document which remains readable (Fig.19) but too strongly degraded for the OCR.

H. S. Baird has already shown that the noise due to the digitization process of document images is located along the stroke contours and fallow a probability law which decreases according to the distance from the contours [51]. He developed a document image model for the
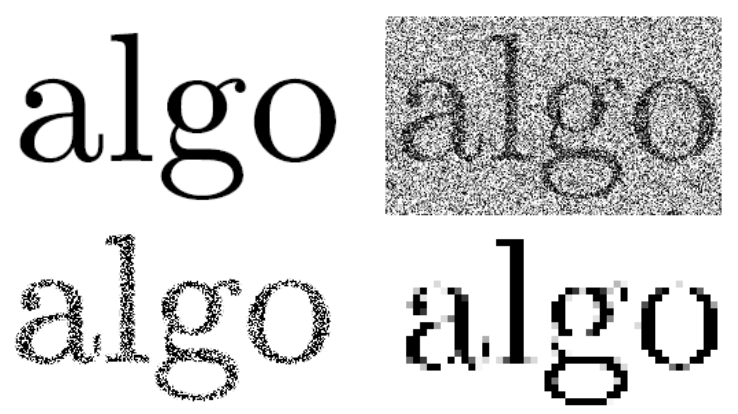

Fig. 19 Distortion in an extract of a document image; the original document image and its distorted versions.

image defects which estimate the degradation of characters around the corners. The model and metrics are mainly for the estimation of classification errors rather than the measurement of visual distortion. For instance, characters degradations are caused by a combination of several effects (digitization, resize, pre-processing, filtering, conversion from a format to another, reduction of color depth, lossy compression...). An adequate measure of document image degradations must also take into account the neighbourhood of pixels and mainly pixels around informative parts which are difficult to locate. Most of the recent works on degradation models for document images show the difficulties to establish a consistent measure of character degradations which is coherent with human perception $[52,53]$. The definition of a quality measure suited for documents images requires deep research works, which is out of the scope of this paper.

In turn, this section takes benefit of the traditional measures (PSNR, MSE) to compare the proposed restoration scheme to the most recent methods in the domain of natural image restoration/denoising. A comparative study is given for two case studies : natural and document noisy images.

\subsection{Natural image denoising}

Initially designed to restore document images, the proposed filter can also be used to denoise natural images. Since the proposed filter is a mixture of the Weickert and the Perona-Malik-Catté diffusion filters, we have the same parameters to fix depending on the image content.

$\sigma$ is the standard deviation of the Gaussian that allows to compute valid gradients (case of Catté). It must be very small; around 0.5 for normal images and until 1.0 for very noisy images.

$\rho$ is the standard deviation of the Gaussian that depends on the level of structures to preserve and reinforce. This value could be modified according to the nature of the structure to preserve and of the lines to reinforce. For the images with small structures and details (Fig.20, Fig.21), $\rho$ must be set to low values (around 1). For im- 


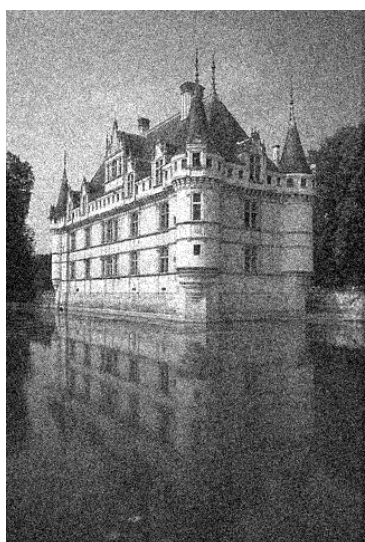

(a) noisy Castle

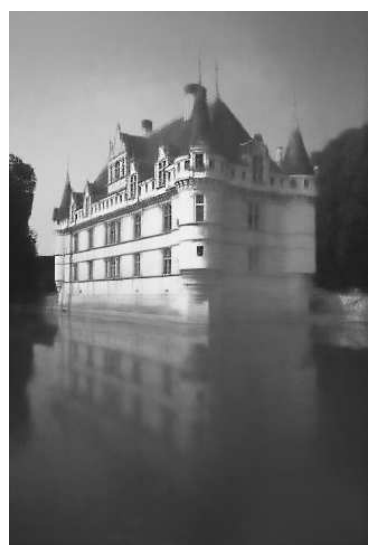

(e) NL Means result

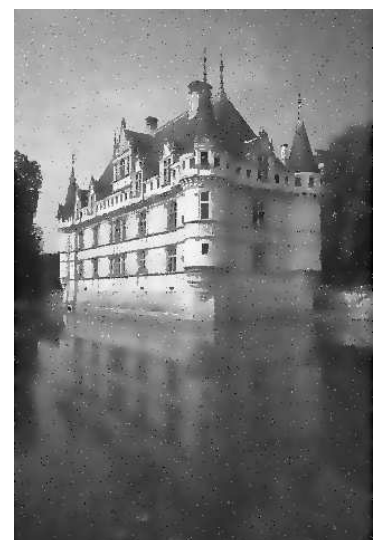

(b) P.-M.-C. result

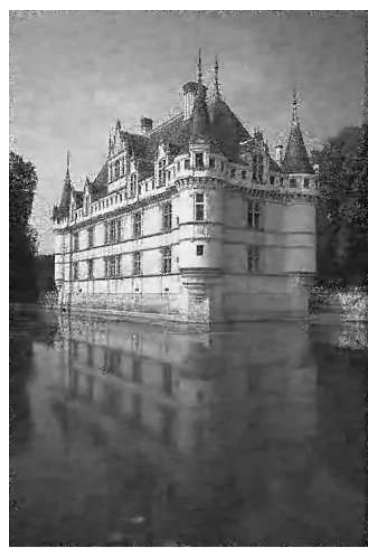

(f) FOE result

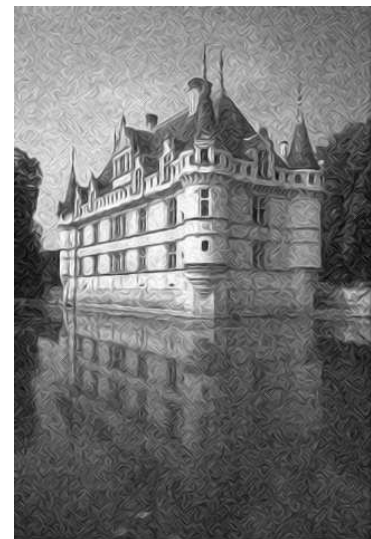

(c) Weickert result

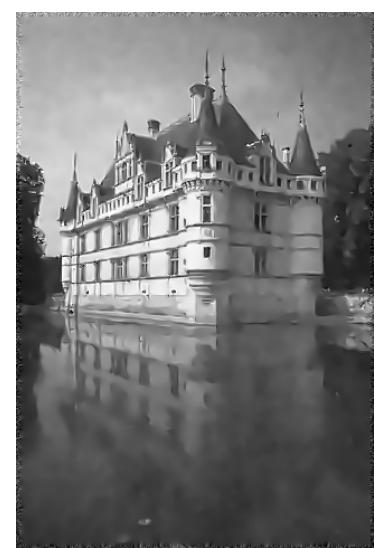

(g) Proposed result

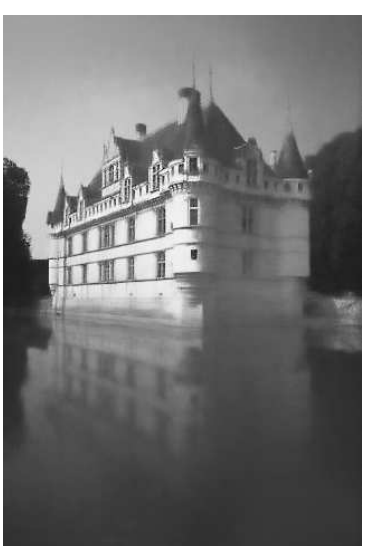

(d) NL Median result

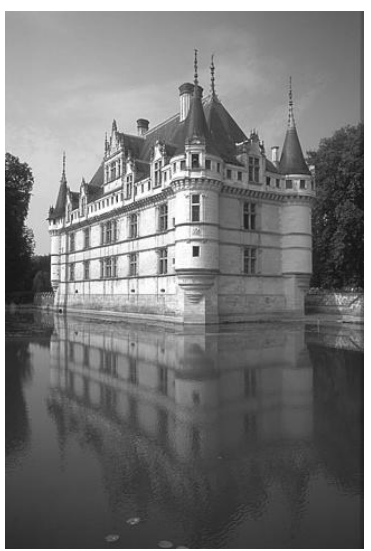

(h) Original image

Fig. 20 A comparative study for image denoising: case of the image Castle.

ages having large and noisy line structures to reinforce (Fig.47), $\rho$ must be set to high values (between 3 to 5 ).

$K_{+}$is the threshold which allows to make a distinction between noise to remove and contours to preserve. Similarly to the Perona-Malik-Catté scheme while processing natural images, $K_{+}$must be set properly according to the level of noise in the image.

$K_{-}$is the additional parameter introduced by the proposed diffusion scheme. This parameter defines the threshold that stops the Weickert coherence effect. For natural images, when $K_{-}$is equal to $K_{+}$, we have a little coherence reinforcement. By increasing $K_{-}$to a value equals to the twice of $K_{+}$, we amplify the weickert effect reinforcing the coherence of lines and repairing discontinuities.

For natural images without additive noise, $K_{+}$is set between 20 and 80 and $K_{-}$is set to $1.5 K_{+}$. In the case of natural images with additive noise, the proposed scheme outperforms the Perona-Malik-Catté, Weickert, NL-means, NL-median restoration models. Only the FOE approach performs a little better.

For instance, a comparative study with different restora-c tion/denoising approaches are conducted on three sam- ples of natural noisy image (Fig.20, Fig.22, Fig.24). A crop and a zoom of these images is given for a better visualisation (Fig.21, Fig.23). The evaluation of the denoising performance relies on three measurements (ISNR, MSE, PSNR). The tables 3,4 and 5 provides these different values. Additional information about the processing time is also given.

In term of speed, the NL-means and FOE require too much time compared to anisotropic diffusion approaches. For instance, the NL-means restoration model reaches a high complexity limit when we increase the windows size $k$ and the search width Maxdist. For the classical image of Barbara $512 \times 512$ with Maxdist equal to 15 , the NL-means model restores in 952" (15m) where as the NL-median model in 7823 " $(2 \mathrm{~h} 10 \mathrm{~m})$. For a better result, when we increase the search width Maxdist to 30, the NL-means model takes 4823" (1h20m) and the NLmedian model 13992" (3h53m). The speed constraints of the NL-means and the FOE approaches make impossible the restoration of document images with high or even with medium resolution. The estimation of the processing time for FOE, NL-mean and NL-median for real documents images of $1000 \times 2000$ is respectively $3 \mathrm{~h}, 10 \mathrm{~h}$, 


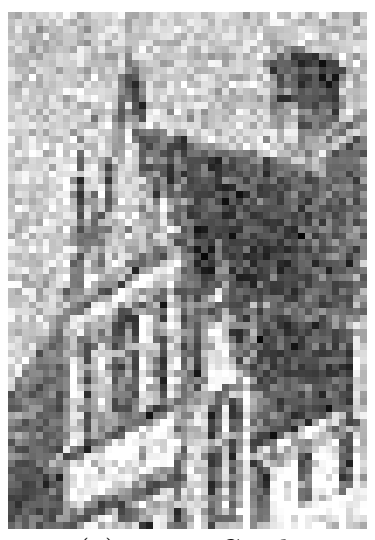

(a) noisy Castle

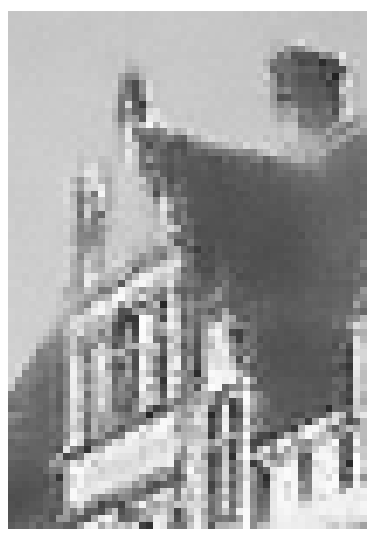

(e) NL Means result

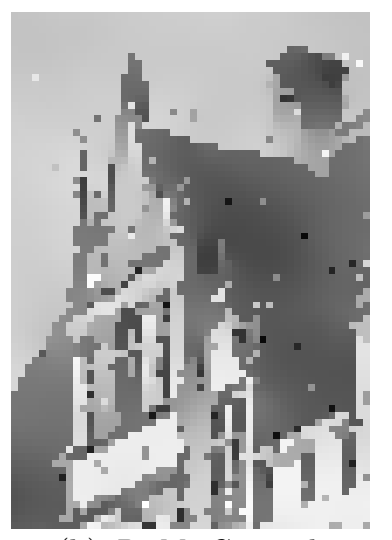

(b) P.-M.-C. result

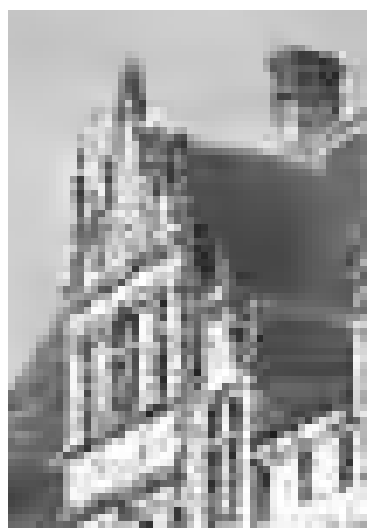

(f) FOE result

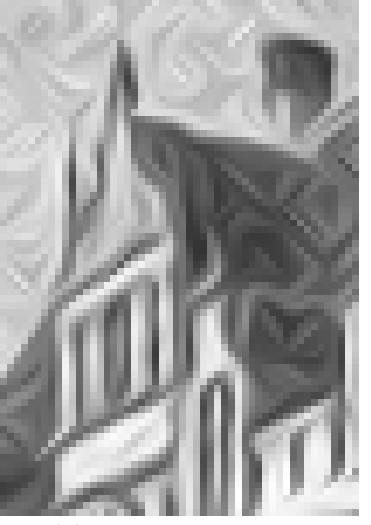

(c) Weickert result

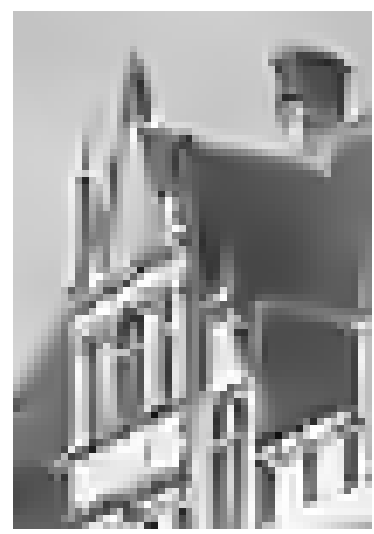

(g) Proposed result

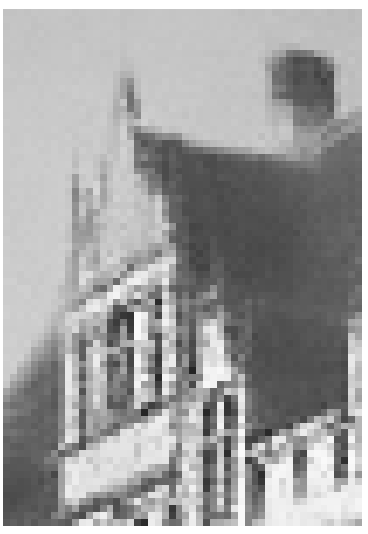

(d) NL Median result

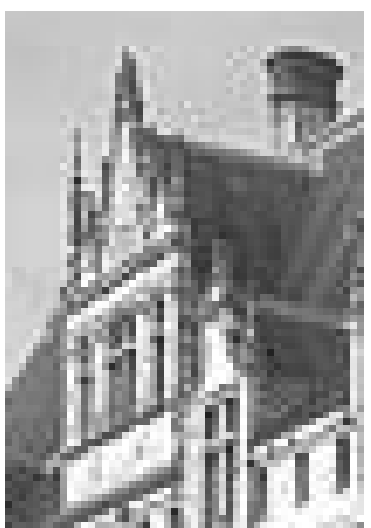

(h) Original image

Fig. 21 A comparative study for image denoising: case of the image Castle; zoom on an extract for a better visualisation.

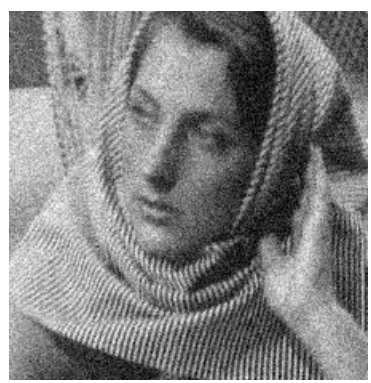

(a) noisy Barbara

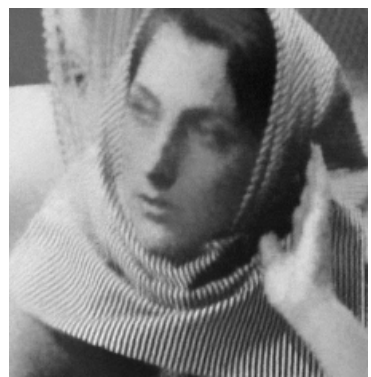

(e) NL Means result

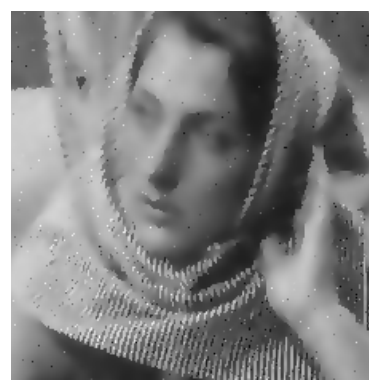

(b) P.-M.-C. result

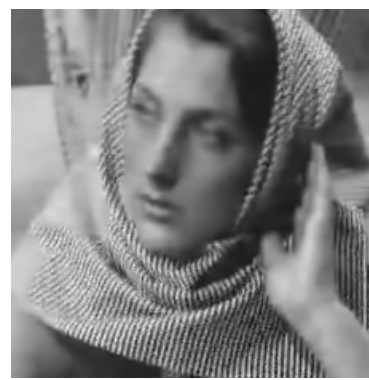

(f) FOE result

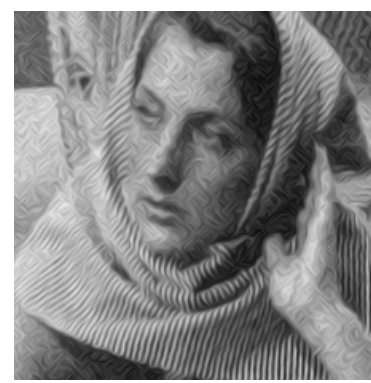

(c) Weickert result

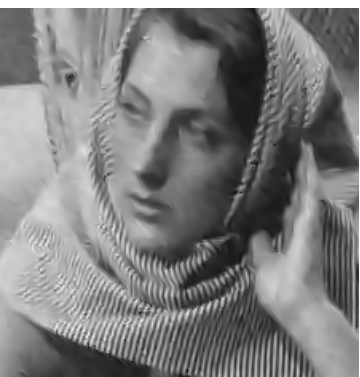

(g) Proposed result

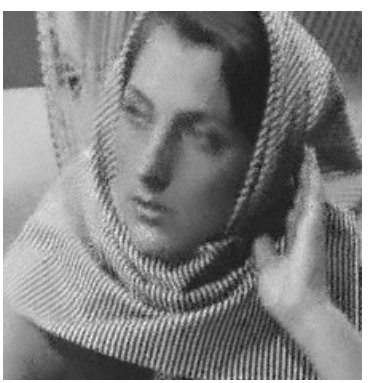

(d) NL Median result

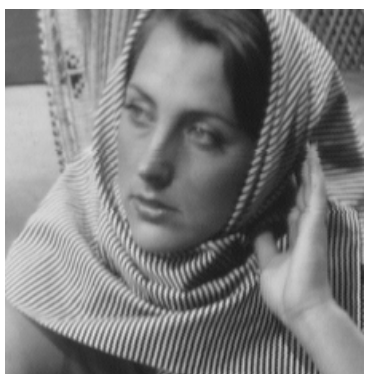

(h) Original image

Fig. 22 A comparative study for image denoising: case of the image Barbara. 


\begin{tabular}{|c|c|c|c|c|c|c|}
\hline & $\begin{array}{c}\text { P.-M.-C. } \\
K=25\end{array}$ & $\begin{array}{c}\text { Weickert } \\
\sigma=0.5 \\
\rho=1.5\end{array}$ & $\begin{array}{c}\text { NL Median } \\
\text { Taxdist }=0.05 \\
\text { Mams } \\
\text { Dims }=80\end{array}$ & $\begin{array}{c}\text { NL means } \\
\text { T=0.05 } \\
\text { Maxdist }=30 \\
\text { Dims }=80\end{array}$ & $\begin{array}{c}\text { FOE } \\
\text { 5X5 cliques } \\
24 \text { filters }\end{array}$ & $\begin{array}{c}\text { Our scheme } \\
\sigma=0.7, \rho=1.0 \\
K_{+}=120 \\
K_{-}=180\end{array}$ \\
\hline \hline ISNR(dB) & +5.1 & +5.2 & +5.2 & +6.4 & +8.3 & +8.0 \\
\hline MSE(dB) & 195.5 & 190.1 & 190.8 & 143.5 & 93.1 & 99.6 \\
\hline PSNR(dB) & 25.2 & 25.3 & 25.3 & 26.5 & 28.4 & 28.1 \\
\hline \hline Time(sec) & $5(+)$ & $6(+)$ & $980(+)$ & $412(+)$ & $2531(+)$ & $25(++)$ \\
\hline
\end{tabular}

Table 3 A comparative study for image denoising: an objective quality measure for the image Castlewith a Gaussian noise $\sigma=25, \quad(M S E=629.1, \quad P S N R=20.1 d B)$ (source: http://www.gris.tudarmstadt.de/ sroth/research/foe/denoising_results.html $)((+)$ Matlab/ $(++) \mathrm{C}++$ /windows $)$

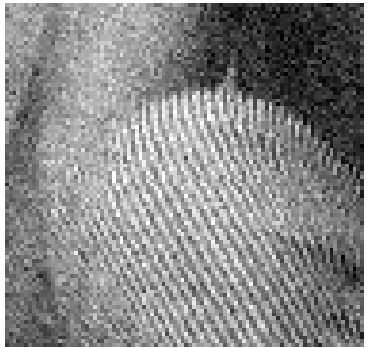

(a) noisy Barbara

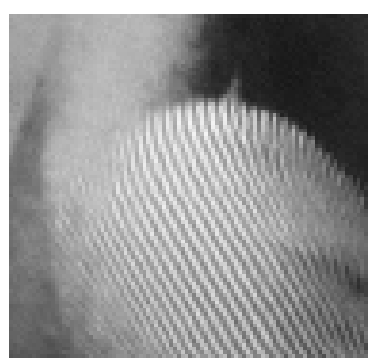

(e) NL Means result

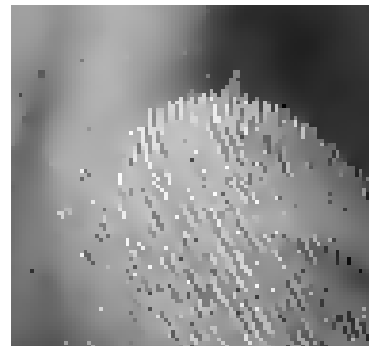

(b) P.-M.-C. result

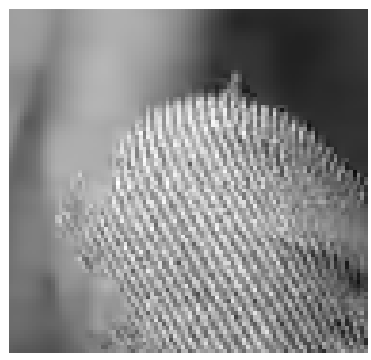

(f) FOE result

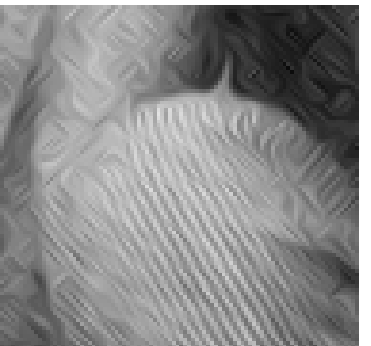

(c) Weickert result

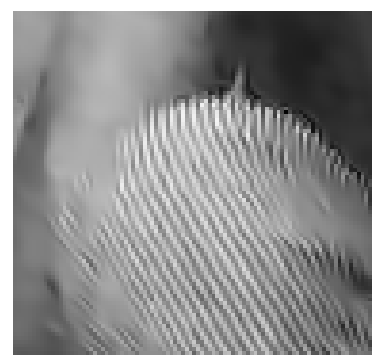

(g) Proposed result

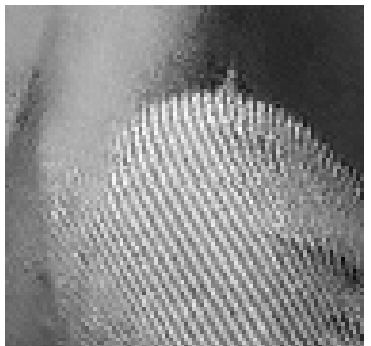

(d) NL Median result

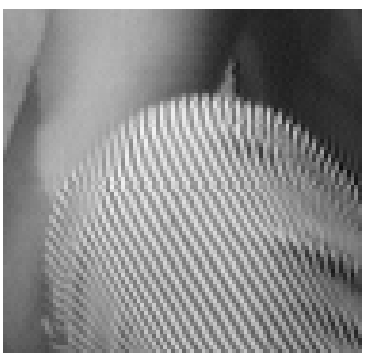

(h) Original image

Fig. 23 A comparative study for image denoising: case of the image Barbara; zoom on an extract for a better visualisation.

\begin{tabular}{|c|c|c|c|c|c|c|}
\hline & $\begin{array}{c}\text { P.-M.-C. } \\
K=25\end{array}$ & $\begin{array}{c}\text { Weickert } \\
\sigma=0.5 \\
\rho=1.5\end{array}$ & $\begin{array}{c}\text { NL Median } \\
\text { Taxdist }=0.03, \\
\text { Dims }=80\end{array}$ & $\begin{array}{c}\text { NL means } \\
\text { T=0.05 } \\
\text { Maxdist }=30 \\
\text { Dims }=80\end{array}$ & $\begin{array}{c}\text { FOE } \\
\text { 5X5 cliques } \\
24 \text { filters } \\
K_{-}=500\end{array}$ & $\begin{array}{c}\text { Our scheme } \\
\sigma=0.5, \rho=1.5 \\
K_{+}=250\end{array}$ \\
\hline \hline ISNR(dB) & +2.8 & +3.5 & +5.3 & +2.6 & +6.2 & +6.0 \\
\hline MSE(dB) & 207.2 & 165.7 & 116.6 & 219.3 & 95.5 & 98.6 \\
\hline PSNR(dB) & 24.9 & 25.6 & 27.4 & 24.7 & 28.3 & 28.1 \\
\hline \hline Time(sec) & $3.9(+)$ & $4(+)$ & $7823(+)$ & $4823(+)$ & $1478(+)$ & $23(++)$ \\
\hline
\end{tabular}

Table 4 A comparative study for image denoising: an objective quality measure for the image Barbara with a Gaussian noise $\sigma=20, \quad(M S E=394.9 d B, P S N R=22.1 d B)$ (source: http://www.gris.tudarmstadt.de/ sroth/research/foe/denoising_results.html $)((+)$ Matlab/ $(++) \mathrm{C}++$ /windows $)$

$24 \mathrm{~h}$ per image. It is the main reason that we compare our scheme in the second part to other anisotropic diffusion schemes ; especially with Perona-Malik-Catté and Weikert from where our approach base its origins.

\subsection{Document image denoising}

For document images, the PSNR values directly extracted from gray level images are not significant, since each restoration approach generates different grayscale images characterized by a background with different gray levels. This is clearly noticeable on the figures 25 and 27 where 


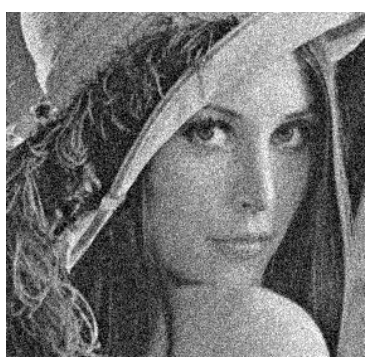

(a) noisy Barbara

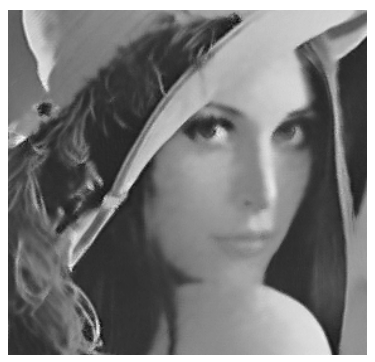

(e) NL Means result

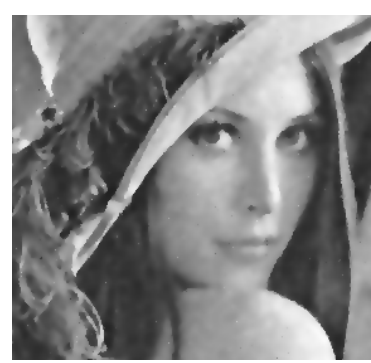

(b) P.-M.-C. result

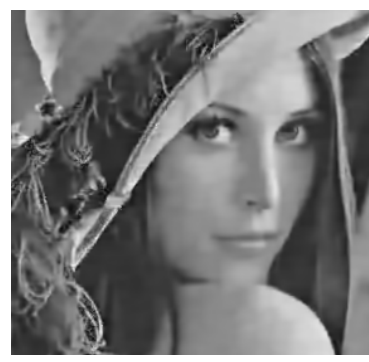

(f) FOE result

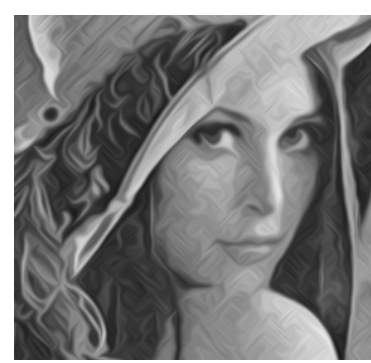

(c) Weickert result

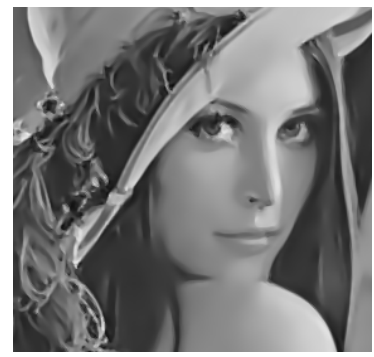

(g) Proposed result

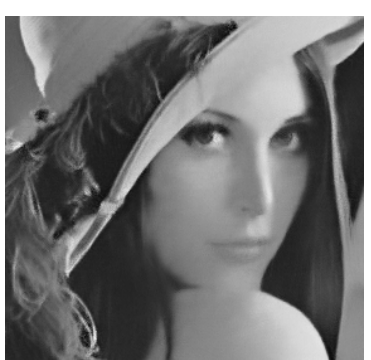

(d) NL Median result

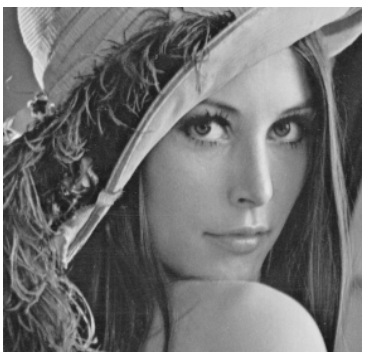

(h) Original image

Fig. 24 A comparative study for image denoising: case of the image Lena.

\begin{tabular}{|c|c|c|c|c|c|c|}
\hline & $\begin{array}{c}\text { P.-M.-C. } \\
K=15\end{array}$ & $\begin{array}{c}\text { Weickert } \\
\sigma=0.5 \\
\rho=1.5\end{array}$ & $\begin{array}{c}\text { NL Median } \\
\text { Taxdist }=0.05 \\
\text { Dims }=80\end{array}$ & $\begin{array}{c}\text { NL means } \\
\text { T=0.05 } \\
\text { Maxdist }=30 \\
\text { Dims }=80\end{array}$ & $\begin{array}{c}\text { FOE } \\
\text { 5X5 cliques } \\
24 \text { filters } \\
K_{-}=375\end{array}$ & $\begin{array}{c}\text { Our scheme } \\
\sigma 0.9, \rho=0.9 \\
K_{+}=250\end{array}$ \\
\hline \hline ISNR(dB) & +3.0 & +9.0 & +6.7 & +8.7 & +10.3 & +10.6 \\
\hline MSE(dB) & 309.0 & 70.5 & 615.4 & 82.6 & 53.2 & 49.5 \\
\hline PSNR(dB) & 23.2 & 29.2 & 26.9 & 28.9 & & 30.8 \\
\hline \hline Time(sec) & $12.1(+)$ & $60(+)$ & $957(+)$ & $4823(+)$ & $1328(+)$ & $72(++)$ \\
\hline
\end{tabular}

Table 5 A comparative study for image denoising: an objective quality measure for the image Lena with a Gaussian noise $\sigma=25, \quad(M S E=615.4 d B, P S N R=20.2 d B)$ (source: http: $/ /$ www.gris.tudarmstadt.de/ sroth/research/foe/denoising_results.html $)((+)$ Matlab/ $(++) \mathrm{C}++$ /windows $)$

\begin{tabular}{|c|c|c|c|c|c|c|}
\hline & $\begin{array}{c}\text { P.-M.-C. } \\
K=25\end{array}$ & $\begin{array}{c}\text { Weickert } \\
\sigma=0.5 \\
\rho=1.5\end{array}$ & $\begin{array}{c}\text { NL Median } \\
\text { Taxdist }=0.2 \\
\text { Dims }=80\end{array}$ & $\begin{array}{c}\text { NL means } \\
\text { Tax }=2.2 \\
\text { Maxdist }=15 \\
\text { Dims }=80\end{array}$ & $\begin{array}{c}\text { FOE } \\
\text { 5X5 cliques } \\
24 \text { filters }\end{array}$ & $\begin{array}{c}\text { Our scheme } \\
\sigma=1, \rho=1 \\
K_{+}=200 \\
K_{-}=300\end{array}$ \\
\hline \hline ISNR(dB) & +11.4 & +11.8 & +8.2 & +10.1 & +12.8 & +12.9 \\
\hline MSE(dB) & 1294 & 1167 & 2648 & 1742 & 924 & 900 \\
\hline PSNR(dB) & 17.0 & 17.4 & 13.9 & 15.7 & 18.4 & 18.5 \\
\hline Cor & 0.9057 & 0.9162 & 0.8272 & 0.8774 & 0.9340 & 0.9357 \\
\hline \hline Time(sec) & $7(+)$ & 13 sec $(+)$ & 765 sec $(+)$ & 480 sec $(+)$ & 576 sec $(+)$ & 75 sec $(++)$ \\
\hline
\end{tabular}

Table 6 A comparative study for image denoising: an objective quality measure for the noisy letter A, $(M S E: 17891 d B$, $P S N R: 5.6 d B)((+)$ Matlab/ $(++) \mathrm{C}++/$ windows $)$

the different measure values are reported in the tables 6 and 8 respectively.

Moreover, the distortions of the character patterns are more important to measure than the regularization of the background. As a solution, we propose to binarize, in a first step, each resulting image with an optimal fixed threshold. As a second step, we extract the different measures from the binary images. We also give the corre- lation cœefficient cor between the restored image and the original one. The latter cœefficient is used to test whether a given test image has been altered. In theory, we would have of value of 1 is the image is intact and a value less than 1 if degradation has occured. Several tests on synthetic images (Fig.25, Fig.26, Fig.27) with different fonts and styles have been achieved. The NL-means, FOE and proposed diffusion scheme perform well compared to the 


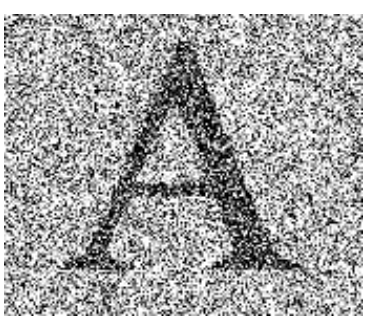

(a) Noisy image

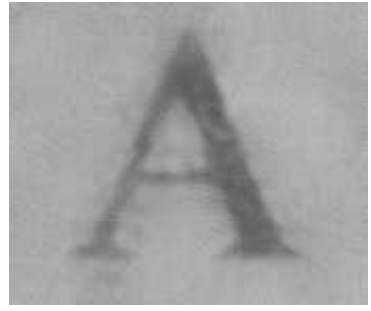

(e) NL Means result

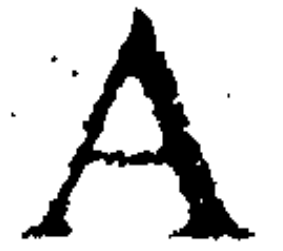

(i) P.-M.-C. result

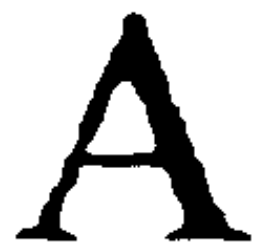

(m) FOE result

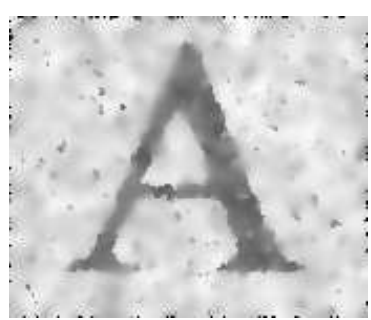

(b) P.-M.-C. result

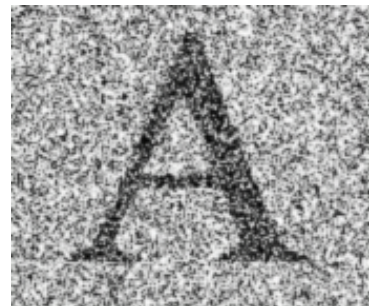

(f) FOE result

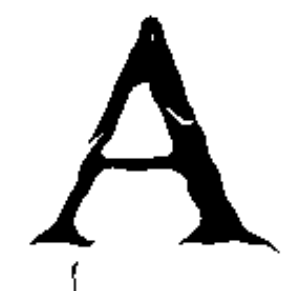

(j) Weickert result

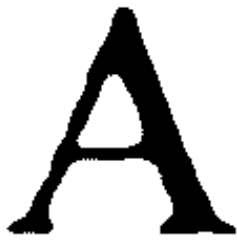

(n) Proposed result

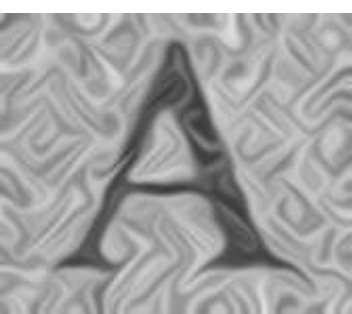

(c) Weickert result

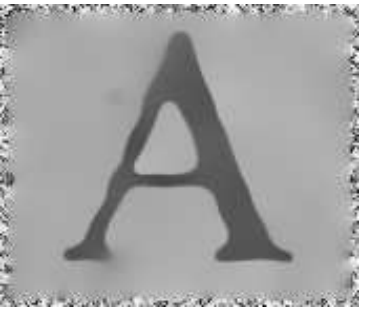

(g) Proposed result

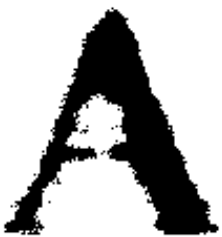

(k) NL Median result

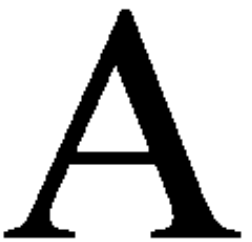

(o) original result

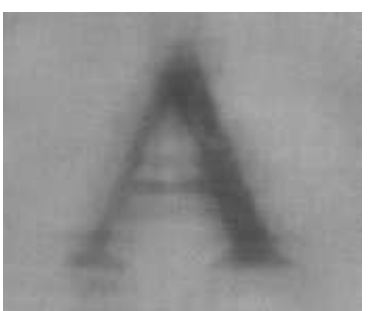

(d) NL Median result

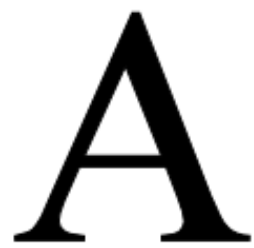

(h) Original text

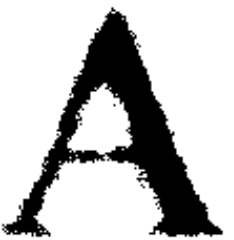

(1) NL Means result

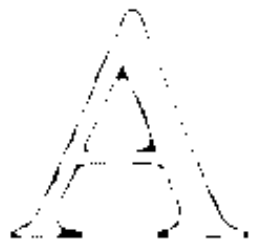

(p) Difference our result/the original

Fig. 25 A comparative study for document image denoising: case of the letter A.

\begin{tabular}{|c|c|c|c|c|c|c|}
\hline & $\begin{array}{c}\text { P.-M.-C. } \\
K=15\end{array}$ & $\begin{array}{c}\text { Weickert } \\
\sigma=0.5 \\
\rho=1.5\end{array}$ & $\begin{array}{c}\text { NL Median } \\
\text { Taxdist=15 } \\
\text { Mims }=80\end{array}$ & $\begin{array}{c}\text { NL means } \\
\text { Dim }=0.05 \\
\text { Maxdist }=30 \\
\text { Dims }=80\end{array}$ & $\begin{array}{c}\text { FOE } \\
\text { 5X5 cliques } \\
24 \text { filters }\end{array}$ & $\begin{array}{c}\text { Our scheme } \\
\sigma=1, \rho=3 \\
K_{+}=200 \\
K_{-}=300\end{array}$ \\
\hline \hline ISNR(dB) & +5.7 & +7.8 & +3.7 & +6.2 & +8.7 & +8.5 \\
\hline MSE(dB) & 1563 & 957 & 2506 & 1410 & 780 & 817 \\
\hline PSNR(dB) & 16.1 & 18.3 & 14.1 & 16.6 & 19.2 & 19.0 \\
\hline Cor & 0.9245 & 0.9557 & 0.87 & 0.93 & 0.96 & 0.96 \\
\hline \hline Time(sec) & $10.2(+)$ & $19(+)$ & $992(+)$ & $596(+)$ & $785(+)$ & $68(++)$ \\
\hline
\end{tabular}

Table 7 A comparative study for image denoising: an objective quality measure for the noisy text Algo, $(M S E: 5881.4 d B$, $P S N R: 10.4 d B)((+)$ Matlab/ $(++) \mathrm{C}++$ /windows $)$ 


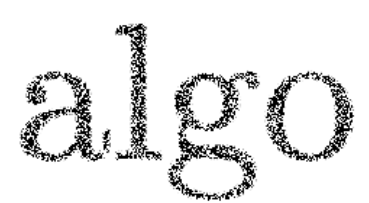

(a) Noisy image

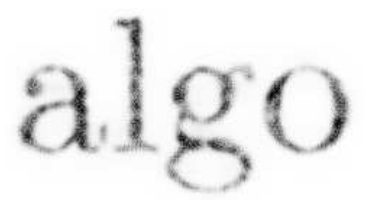

(e) NL Means result

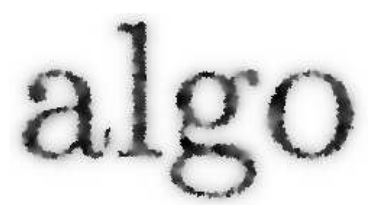

(b) P.-M.-C. result

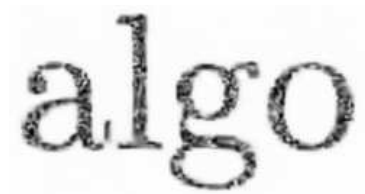

(f) FOE result

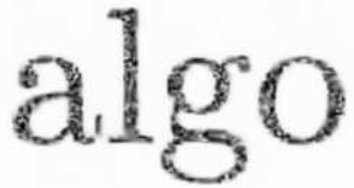

(c) Weickert result

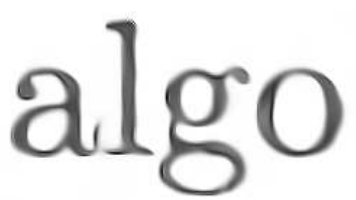

(g) Proposed result

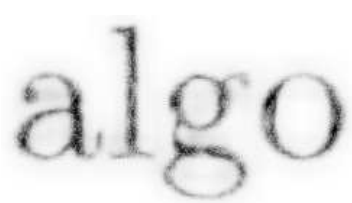

(d) NL Median result

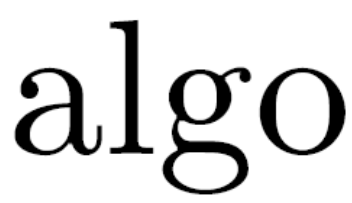

(h) Original text

Fig. 26 A comparative study for document image denoising: case of the text Algo.

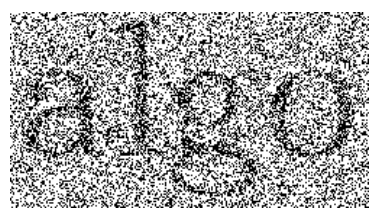

(a) Noisy image

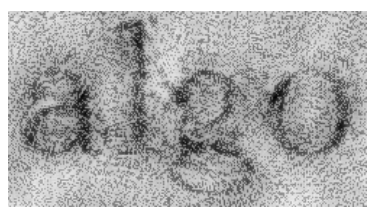

(e) NL Means result

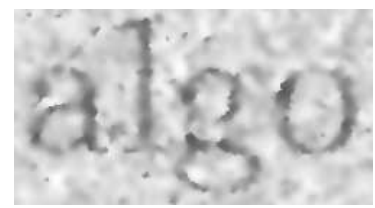

(b) P.-M.-C. result

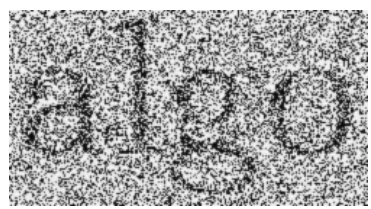

(f) FOE result

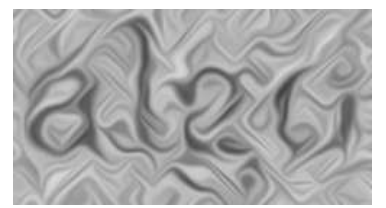

(c) Weickert result

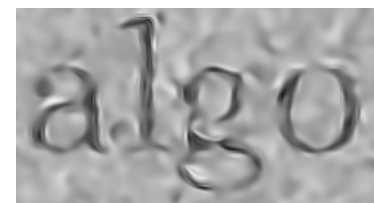

(g) Proposed result

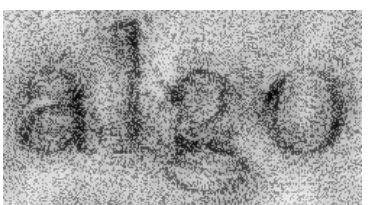

(d) NL Median result

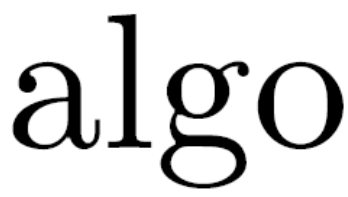

(h) Original text

Fig. 27 A comparative study for document image denoising: case of the text Algo with a noisy background.

\begin{tabular}{|c|c|c|c|c|c|c|}
\hline & $\begin{array}{c}\text { P.-M.-C. } \\
K=25\end{array}$ & $\begin{array}{c}\text { Weickert } \\
\sigma=0.5 \\
\rho=3\end{array}$ & $\begin{array}{c}\text { NL Median } \\
\text { Taxdist }=15 \\
\text { Dims }=80\end{array}$ & $\begin{array}{c}\text { NL means } \\
\text { T=0.3 } \\
\text { Maxdist }=15 \\
\text { Dims }=80\end{array}$ & $\begin{array}{c}\text { FOE } \\
\text { 5X5 cliques } \\
24 \text { filters }\end{array}$ & $\begin{array}{c}\text { Our scheme } \\
\sigma=1, \rho=3 \\
K_{+}=200 \\
K_{-}=300\end{array}$ \\
\hline \hline ISNR(dB) & +7.9 & 6.3 & +4.9 & +5.0 & +0.05 & +9.0 \\
\hline MSE(dB) & 3367 & 4772 & 6713 & 6587 & 20484 & 2593 \\
\hline PSNR(dB) & 12.8 & 11.3 & 9.8 & 9.9 & 5.0 & 13.9 \\
\hline Cor & 0.8056 & 0.70 & 0.52 & 0.54 & 0.22 & 0.84 \\
\hline \hline time(sec) & $15.5(+)$ & $28(+)$ & $1461(+)$ & $801(+)$ & $513(+)$ & $92(++)$ \\
\hline
\end{tabular}

Table 8 A comparative study for image denoising: an objective quality measure for the noisy text Algo, $(M S E: 20764 d B$, $P S N R: 4.9 d B)((+)$ Matlab/ $(++) \mathrm{C}++$ /windows $)$

Perona-Malik-Catté and the Weickert diffusion filter. For instance, the correlation values associated to each case study and given in the tables 6,7 and 8 illustrate the efficiency of the proposition filter ; for all the case studies, the corresponding correlation values is superior to all the others. In fact, the document images and mainly binary ones are too much contrasted along all the characters contours. In this case, the Perona-Malik-Catté process could not work correctly because the diffusion process stop too early near the contours of these document image characters.
The matrix driven diffusion model of Weickert performs generally better than the Perona-Malik-Catté model but the reinforcement of the coherence by the Weickert diffusion filter tends to modify the topology of characters. According to the given results, the proposed diffusion filter is well suited for document images since it takes benefit of the Weickert coherence reinforcement while stopping this process around singularities like corners. With low noise level, NL means, FOE and the diffusion filters restore correctly with almost the same ISNR. The difference of the behavior statement between all the restoration models is noticeable in the case of 
severely degraded images and especially when adding a large amount of noise (Fig.25 ). Faced with such situation, the performances of the NL-means filter decreases quickly. The FOE and the proposed scheme outperform all the other restoration models.

Figure 25 shows that without noise in the background, the different given approaches perform globally in a good manner. For document images, the presence of noise in the background has a great impact on the overall performance of the restoration process. The FOE and the proposed scheme achieve again better results and remains almost stable.

We have also tested the limits of all the restoration models in the case of excessive noise (Fig.27). Restoration approaches based on PDE remain more efficient compared to the others. For images with excessive noise, the NL means and the FOE filters fail in restoring the visual degradation.

For the diffusion based restoration approaches and the FOE filter, the computation cost depends on the number of iterations. Thus, we stop the iteration process when the PSNR improvement does not change. The Perona-Malik-Catté and the Weickert restoration filters require only 20 to 50 iterations. The proposed restoration scheme requires between 50 and 200 iterations depending on the noise level. The FOE filter needs between 1500 and 3000 iterations to reach optimal results. Concerning the NL-means filter, it requires too much computation time to be used on document images. By taking optimal parameters with larger search window size and larger template size, NL-means performs a little better but the computational cost increases exponentially. Without optimization, NL-means and FOE approaches cannot be used on large images like document images.

\section{Experimental results : Application for degraded document image restoration}

\subsection{Visual quality improvement}

To illustrate the performance of the proposed diffusion filter, we give some experimental results on real document images.

A first test image is an extract of a degraded character image provided from the database of "British Libraries" (Fig.28). We clearly notice a good visual improvement results four the noisy original image. The restored image reveals the efficiency of the proposed anisotropic diffusion process. For instance, we notice an isotropic smoothing in homogeneous regions, removing efficiently the noise and avoiding undesired image structure appearance. The diffusion preserves vector edges and it is well studied on sharp corners as it prevents corner erosion. More tests with a noticable visual quality improvement are shown respectively in figure 29 and figure 30 .

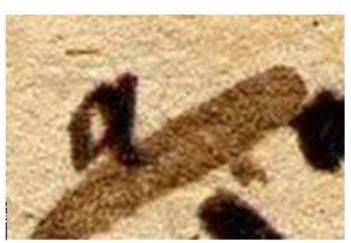

(a) Original image

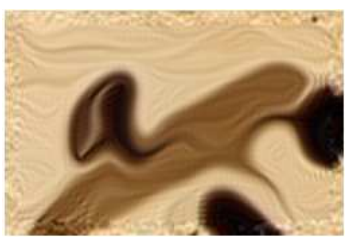

(c) Wei. diffusion filter

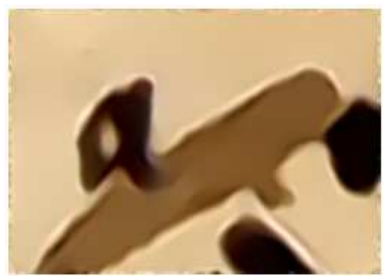

(e) Tsch. diffusion filter

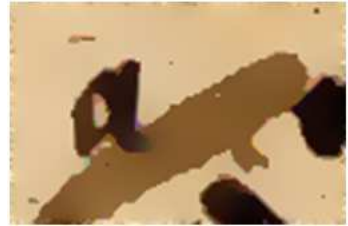

(b) P.-M. diffusion filter

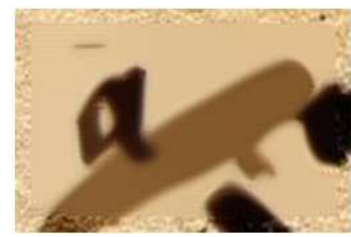

(d) Belt. diffusion filter

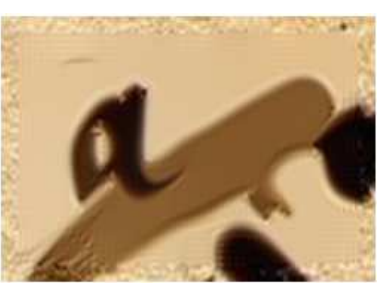

(f) Our diffusion filter
Fig. 32 Results of the application of different diffusion filters on a degraded character extracted from a document provided by the archive of "Chatillon-sur-Chalaronne".

Moreover, the proposed filter is not confined to the processing of textual documents; other kinds of images could also be processed. We give for example results on graphical printed images generated after the application of the different diffusion filters (Fig.31). For instance, we test, beside the proposed diffusion filter, the diffusion filters of Perona-Malik-Catté (Fig.31(b)), Alvarez (Fig.31(c)), Weickert (Fig.31(d)) and Terebes (Fig.31(e))

The diffusion filter of Terebes is given by

$$
\left\{\begin{array}{l}
I_{t}=\operatorname{div}\left(C\left(\left\|G_{\sigma} \otimes \nabla I\right\|\right) \times\left(\begin{array}{c}
I_{x} \cos \theta \\
I_{y} \sin \theta
\end{array}\right)\right) \\
I_{0}=\quad I_{\text {Noisy }}
\end{array}\right.
$$

Other experiments have been done in order to compare the performance of our diffusion filter with its basic approaches applied separately and even with other known diffusion processes. For instance, we test these approaches on a degraded character extracted from a document provided by the archive of "Chatillon-sur-Chalaronne" (Fig.32).

The application of typical nonlinear diffusion methods on degraded document image characters reveals that anisotropic diffusion is well-suited for the processing of the oriented features. For instance, diffusion processes driven by anisotropic tensors include edge enhancing diffusion that tends to preserve important features with 

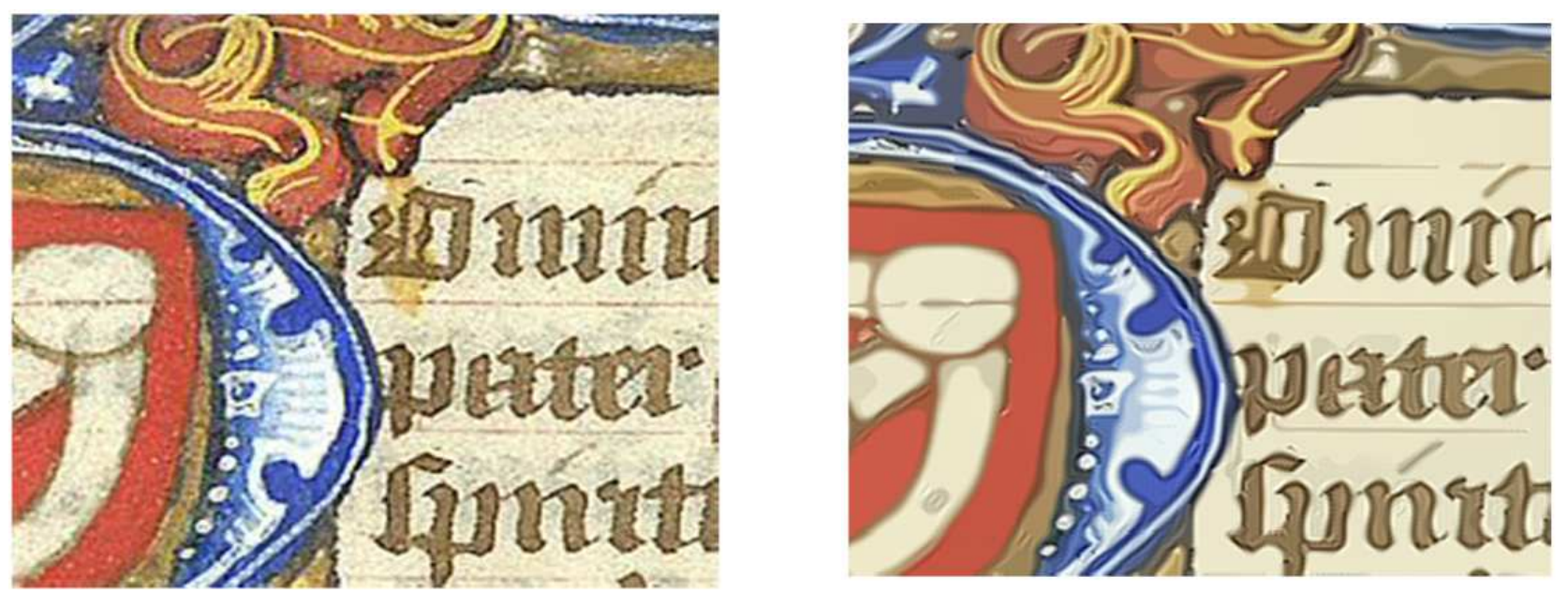

Fig. 28 From left to right respectively : Original image (source British Libraries) and its restored version with our proposed diffusion filter for $\sigma=0.5, \rho=0.5, K_{+}=20, K_{-}=80$.
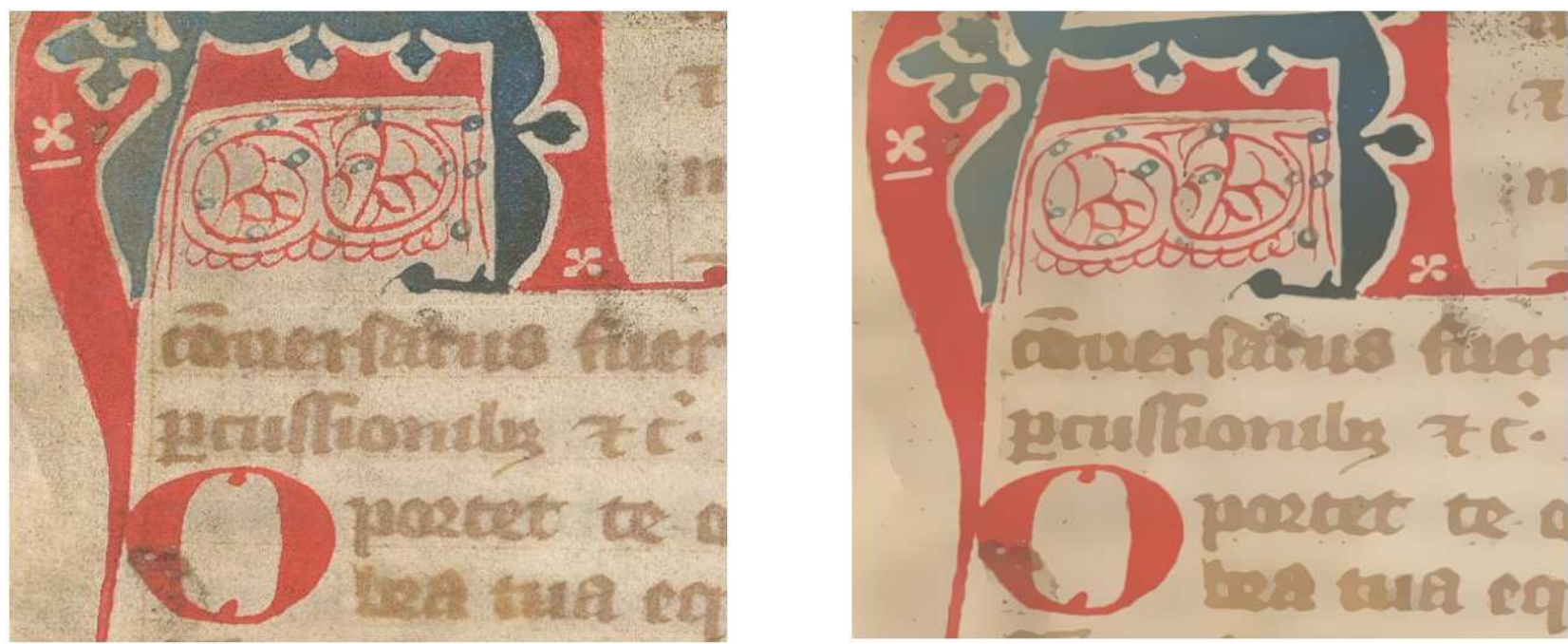

Fig. 29 From left to right respectively : Original image (source IRHT) and its restored version with our proposed diffusion filter.
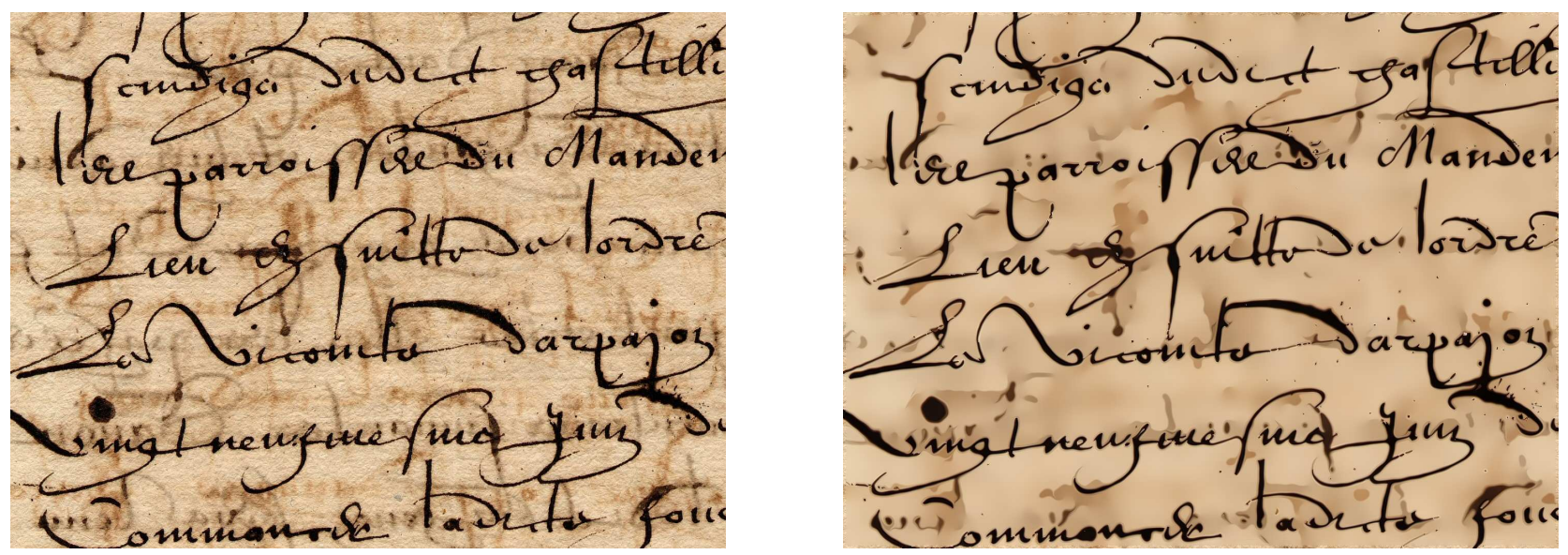

Fig. 30 From left to right respectively : Original image (Châtillon-sur-chalaronne) and its restored version with our proposed diffusion filter. 


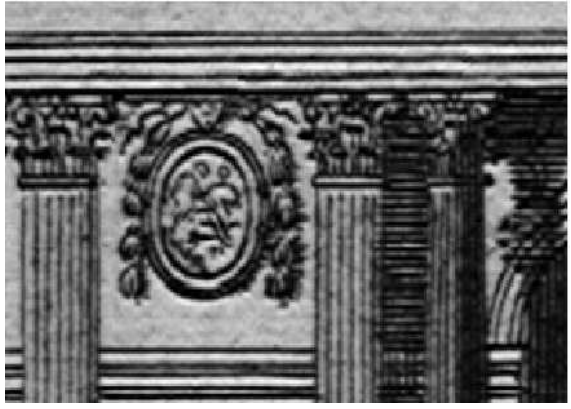

(a) Original image

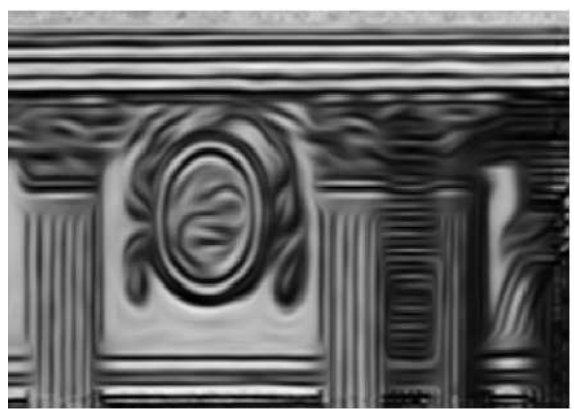

(d) Weickert diffusion filter

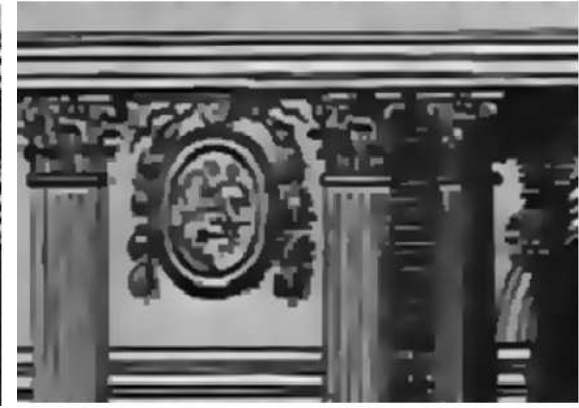

(b) Perona-Malik-Catté diffusion filter

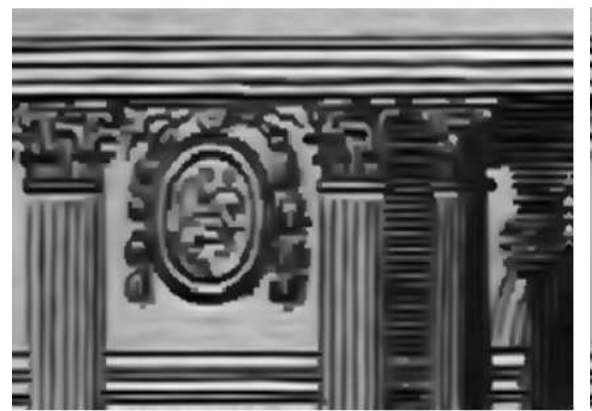

(e) Terebes diffusion filter

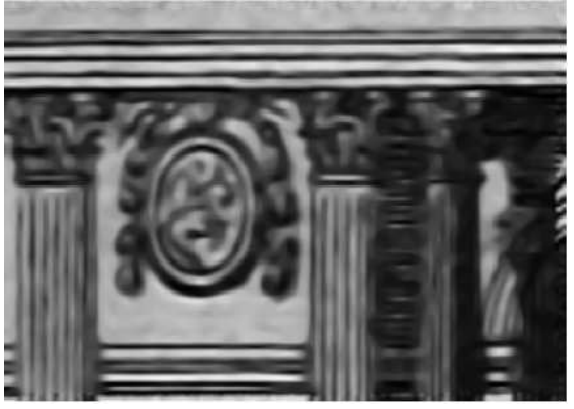

(c) Alvarez diffusion filter

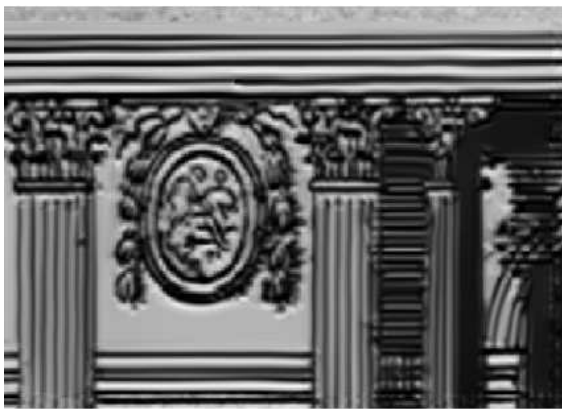

(f) The proposed diffusion filter

Fig. 31 Comparison of different diffusion filters on a printed graphical image.

noise removal during smoothing and coherence-enhancing diffusion that processes the oriented structures by smoothing along the flow direction. In general, better preservation of image details is achieved with the new tensor diffusion filter (Fig.31).

Low resolution and the compression with a lossy compression like JPEG generate many artefacts in document images, mainly those with very small typefaces like old journals [55]. Consequently, document characters are aliased and difficult to be read. As a solution, we propose to treat such degradations by resizing without interpolation (pixel resized) the image with a factor of 4 followed by the application of PDE diffusion filters on the resized image. Figure 33 shows the resized image (Fig.34(a)) and the restored resized image by the proposed PDE filter (Fig.34(b)). In this case, our filter is used to magnify the image, reduce noise, restore character shapes and remove JPEG artefacts. We visually compare results with this magnification process with those proposed by D. Tschumperlé [50]. We use Greystoration version 2.9 from the same author with default parameters and we deactivate the sharpening process to avoid undesirable effects. The magnification results generated with Greystoration tools (Fig.34(c)) reveal a little more aliasing effects. A crop and a zoom of the processed images is given in the figure 34 pour a better visualisation.

The evaluation of the diffusion filters has been conducted in a visual way by comparing the visual quality of each studied image before and after diffusion. The next section will give a quantitative measure by studying OCR (without syntactical analysis and post-processing) results on printed original/restored degraded document images available on-line by the most popular digital libraries.

\subsection{OCR accuracy improvement}

While the evaluation of natural image quality after a processing step with a PDE filter is possible thanks to standard measures such as SNR (Signal to Noise Ratio) or MSE (Mean Square Error), it is not the case with degraded textual document images. In fact, these global measurements are more quantitative rather than qualitative. They do not take the importance of information along characters into account. We can give a small amount of noise located on the part that distinguishes the letter "t" from the letter "l" as an example. The value of the SNR close to 0 in this case gives the illusion that the image is not degraded but in reality this is not true as this little modification has changed the meaning. Thus, the evaluation of the quality of restored textual document images is not an easy task. There exists no general methodology to evaluate the correctness of a given result. Since the ultimate aim of our PDEalgorithm is to restore damaged characters in noisy document images, we propose to evaluate the result through the study of the optical character recognition accuracy rate. An improved recognition rate is certainly related in 
Fête d la bawrse.

Londres, 26 octobre.

Nous apprepous de Gibraltar, da 5 octo forté de trois frégates, quatre sloops aperçue du cóté de l’Occident: Les vaissean? ne portäiéte aucur pavilton. I] est vraiser

(a) Case of pixel resized image with a factor 4 on image [55]

Fête d la bourse.

\section{Londres, 26 ocsobre.}

Nious apprenons de Gibraltar, du 5 octo forte de trois frégates, quatre sloops aperçue du còté de l'Occident. Les vaisseau: ne portaient aucun pavillon. Il est rraiset

(b) Case of a magnification with our proposition with a factor 4

Fète i la hourse.

\section{Londres, 26 octobre.}

Nous apprenons de Gibraltar, du 5 octo forte de trois frégates, quatre sloops aperçue du côté de l'Occident. Les vaisseau: ne portaient aucun pavillon. Il est rraiser

(c) Ċase of a magnification with Tschumperlé restoration process with a factor 4 


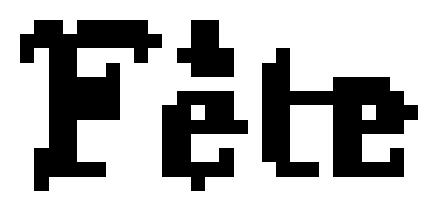

(a) Original image [55]

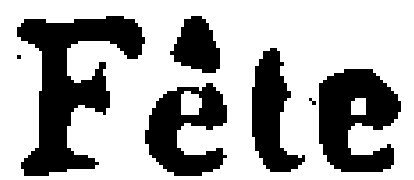

(b) Proposed filter result

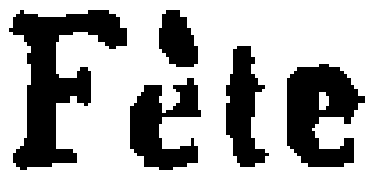

(c) Tschumperlé filter result

Fig. 34 Application of the magnification processes with a factor 4 on a zoom of the test image provided by [55].

this case to an improved quality image. Thus, this section focuses on studying OCR system response before and after restoring damaged characters with PDE-based approaches. This study will be with the same parameters and the same OCR package. ABBYY Finereader 8.0 professional Edition is the OCR software widely known for its high precision character recognition. Because modern OCR cannot recognize obsolete old letters, we do not count classical errors with the confusion between long "s" and "f" for example.

To prove the generality of our proposition, we choose historical printed books and old newspapers of the 16-18 century which are particularly degraded. These resources are available to all researchers on popular on-line digital libraries like Gallica or Google books. Since we participate in the digitization of the "Gazettes of Leydes", we include these documents showing degradations explained by the documents aging and the bad quality of the printing.

For all these document images, we give the recognition rate of the OCR before and after restoration. We count manually the number of characters which are not recognized before $(N b)$ and after $(N a)$ the restoration. By comparing the recognition rates, we cannot measure precisely how many characters are improved or degraded by the restoration. For a precise evaluation, we also count the number of improved characters $(N i)$ and the number of degraded characters $(N d)$ by the restoration process. We reach our purpose if the number of improved characters $(N i)$ is greater than the number of degraded characters $(N d)$ by the restoration process. In this case, $N i-N d$ is positive and represents the number of characters correctly recognized after the restoration. To avoid errors during the counting, we have checked for all documents that $N a-N b=N i-N d$.

\subsubsection{Case of Gallica document images}

Gallica digitizes degraded microfilms instead of the original books in order to reduce the cost and increase the speed of the digitization process. Most of the images are binary compressed with a lossy compression. The resolution is enough for the OCR but the loss of gray scale information, the defects of microfilms and the bad dig- itization process make the character segmentation and recognition very difficult. A large number of pages are empty or unusable because of the lack of control of the image quality. The restoration of these images is very beneficial for the OCR especially when the noise around the contours and in the background makes the segmentation and the recognition of characters difficult.

The book titled "Le bourgeois gentilhomme" from Molière [56], printed in 1671, presents severe degradations due to the digitization process affecting the image quality with random dithering and replacing lines by isolated dots. Figure 35 details the effect of the restoration on an extract of an image ; the corresponding extracts of the OCR results obtained on the different document images are also given. A crop and a zoom of the different processed images is introduced for a better visualisation (Fig.36).

This good visual improvement of the degraded document image after the application of our filter is accompanied by a good recognition rate shown in tables 9 and 10. Actually, before processing the initial document image given in figure 35.a, the OCR system completes the conversion with the accuracy rate of $85.93 \%$. Thus, a $14.07 \%$ failure rate is resulting. According to the restored image 35.b, the OCR system had approximately a $94.47 \%$ success rate when detecting damaged letters. This rate is noticeably higher than the recognition rates calculated on the original non-processed image. The accuracy of the OCR system was well improved by processing a document page with the proposed filter and we succeed in decreasing the failure rate to $5.53 \%$. We have also reported that $1.62 \%$ of the characters have been degraded and $10.16 \%$ have been improved by the restoration. We notice that the OCR fails to segment correctly the characters when they have too many discontinuities. The restoration also degrades some characters like the letter "N" in the first line or "e" in the last line (Fig.35). These reconstruction errors are explained by the disorientation of the flow explained above.

The second book [57], printed in 1787, is a priori the less degraded documents we have collected. Figure 38 shows minor differences between the original (Fig.38.a) and the restored image (Fig.38.b). A little improvement is thus achieved. Nevertheless, the restoration improves 
de faire imprimer, vendre \& debiter une Piece de Theatre, intitulé LE BOURGEOIS: GENTILHOMME, par tel lmprimeur.

(a) Original degraded image

défaire imprimer, vendre $8 \mathrm{t}$ débiter une Pièce ${ }^{1}$

de Théâtre, intitulée LE B-OURGEOIS-

CE NT I L H O M M-S , par tel Imprimeur.

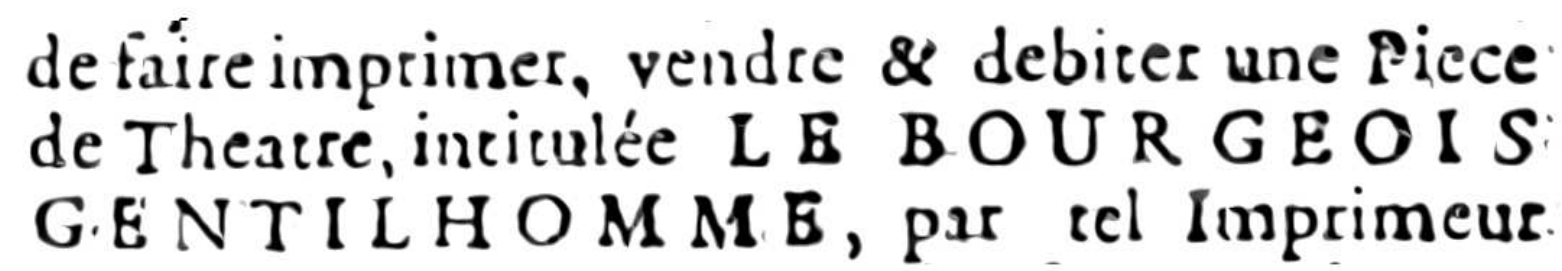

(b) Image restored with the proposed diffusion filter

de faire imprimer, vendre Si débiter une Pièce

de Théâtre, intitulée LE BOURGEOIS

G E N T I L H O M M E, par tel Imprimeur

\section{defaire inprimer, vendre \& debiter une piece de Theatre, intitulée L B BOUR GEOIS GENTILHOMME, par tel Imprimeur}

(c) Image restored with the Perona-Malik-Catté diffusion filter

de taire imprimer, vendre Se dé biter une Pièce

de Théâtre, intitulée LE BOURGEOIS

G $\Sigma$ N T I L H O M M I, par tel Imprimeur

defaire imprimer, vendre \& debiter une Riece
de Theatre, intitulé L E BOUR GEOIS
G.ENTILHOM ME, par tel Imprimeus

(d) Image restored with the Weickert diffusion filter

de faire imprimer, vendre Si dé biter une Pièce

de Théâ tre, intitulé e L H BOURGEOIS

G E NT I L H O M M B , par tel Imprimeur,

Fig. 35 Details of the image N0070212-TIFF-1-20 [56] before and after restoration with the proposed filter, the PeronaMalik-Catté filter and the Weickert diffusion filter. Small extracts of the OCR results obtained on the different document images are also given. 

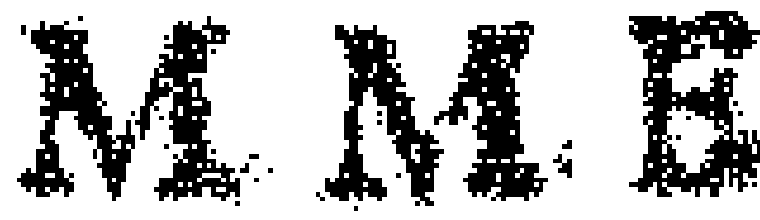

(a) Extract of the Original degraded image
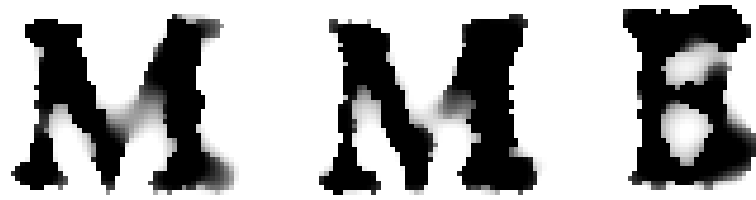

(c) Extract of the image restored with the P.-M.-C. filter
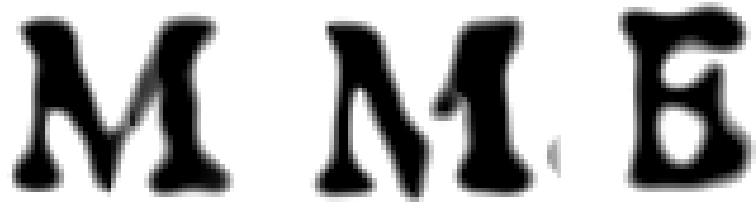

(b) Extract of the image restored with our filter
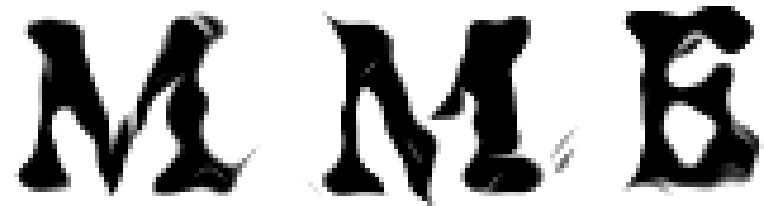

(d) Extract of the image restored with the Wei. filter

Fig. 36 Zoom on extracts of the image N0070212-TIFF-1-20 [56] before and after restoration with the proposed filter, the Perona-Malik-Catté filter and the Weickert diffusion filter.

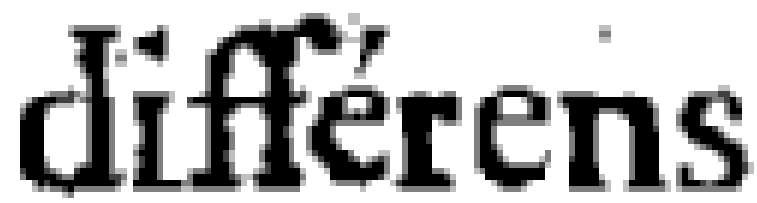

(a) Extract of the Original degraded image

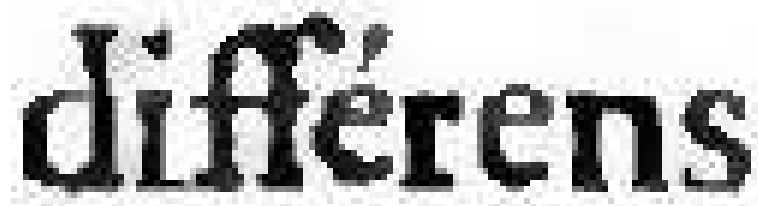

(c) Extract of the image restored with the P.-M.-C. filter

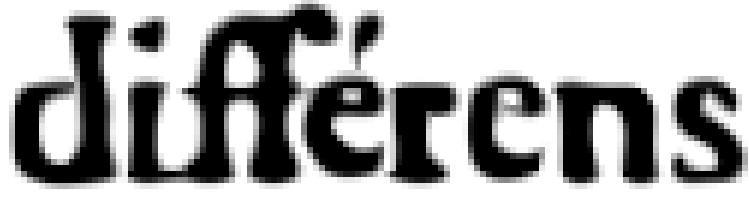

(b) Extract of the image restored with our filter

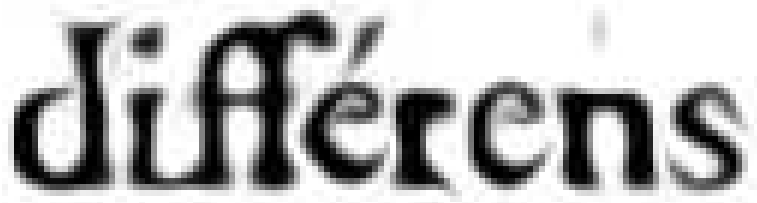

(d) Extract of the image restored with the Wei. filter

Fig. 37 Zoom on extracts of the image N001157109-2 [57] before and after restoration with the proposed filter, the PeronaMalik-Catté filter and the Weickert diffusion filter.

\begin{tabular}{|c|c|c|c|c|}
\hline & Fig.35[56] & Fig.38[57] & Fig.41[54] & Fig.??[59] \\
\hline \hline Total number of characters & 1230 & 781 & 705 & 777 \\
\hline Number of errors before restoration & 173 & 30 & 192 & 170 \\
\hline $\begin{array}{c}\text { Number of errors after } \\
\text { restoration with our filter }\end{array}$ & 68 & 13 & 128 & 68 \\
\hline $\begin{array}{c}\text { Number of errors after } \\
\text { restoration with the Weickert filter }\end{array}$ & 69 & 19 & 127 & 84 \\
\hline $\begin{array}{c}\text { Number of errors after } \\
\text { restoration with the P.M.C. filter }\end{array}$ & 107 & 21 & 150 & 164 \\
\hline
\end{tabular}

Table 9 Impact of the restoration on the total number of errors; a study with three different PDE-based approaches (the proposed filter, the Weickert filter and the P.M.C. filter) on different document images.

the OCR accuracy from $94.57 \%$ to $98.23 \%$ (Tab.9). It shows that the performance of OCR is unpredictable due to its complexity and to the implication of numerous heuristics controlled by different parameters estimated automatically. Consequently, documents which appear sufficiently clean could also be preprocessed by the OCR for a better result; even a little enhancement may have a great impact on the OCR.

For the book of Nostradamus [54], printed in 1589, figure 41 shows the result of the restoration and the preservation of the character shapes during the noise removal in the background. The recognition rate rises from $94.2 \%$ to $98.2 \%$ after restoration with only $0.7 \%$ of char- 


\begin{tabular}{|c|c|c|c|c|}
\hline $\begin{array}{c}\text { Figure } \\
\text { Number }\end{array}$ & $\begin{array}{c}\text { \% of Improved } \\
\text { characters }\end{array}$ & $\begin{array}{c}\text { \% of degraded } \\
\text { characters }\end{array}$ & $\begin{array}{c}\text { \% recognition before } \\
\text { restoration }\end{array}$ & $\begin{array}{c}\text { \% recognition after } \\
\text { restoration }\end{array}$ \\
\hline \hline Fig.35[56] & $10.16 \%$ & $1.62 \%$ & $85.93 \%$ & $94.47 \%$ \\
\hline Fig.38[57] & $2.68 \%$ & $0.76 \%$ & $96.15 \%$ & $98.33 \%$ \\
\hline Fig.41[54] & $4.69 \%$ & $0.76 \%$ & $72.76 \%$ & $81.84 \%$ \\
\hline Fig.42[59] & $2.69 \%$ & $0.71 \%$ & $96.80 \%$ & $98.78 \%$ \\
\hline Fig.44[59] & $6.18 \%$ & $5.22 \%$ & $93.81 \%$ & $94.77 \%$ \\
\hline
\end{tabular}

Table 10 Impact of the restoration with the proposed PDE-based approach on the recognition rate of different document images.

noble tâche qu'il s'étoit imposée, après avoir développé tous Ies rapports qui existent entre les différens Etats de l'Europe,

(a) Original degraded image

\section{noble tâche qu'il s'ćtoit imposée, après avoir développé tons Ics rapports qui exis- tenc entre les différens Erats de l'Europe,}

(b) Image restored with the proposed diffusion filter

noble tâche qu'il s'étoit imposée, après avoir développé tous Ics rapports qui existent entre les différens Etats de l'Europe,

(c) Image restored with the Perona-Malik-Catté diffusion filter

\section{noble tâche qu'il s'ćtoit imposée, après avoir développé tous Ics rapports qui exis- tent entre les différens Erats de l'Europe,}

(d) Image restored with the Weickert diffusion filter

Fig. 38 Details of the image N001157109-2 [57] before and after restoration with the proposed filter, the Perona-Malik-Catté filter and the Weickert diffusion filter. Small extracts of the OCR results obtained on the different document images are also given. 
considérables, et que de l'emploi de ces capitaux naissent les richesses qui tendent toujours à s'augmenter, il eft démontré que l'inégalité dans les; fortunes découle nécessaircment de la propriété; ; il est prouvé que, de même, qu'il existe des

(a) Original degraded image

Xonsîdérables, et que de l'emploi de ce*

...capitaux naissent les richesses qui tendent

toujours s'augmenter, il eft démontré

.nque Finégalité dans les, fortunes découle"

nécessairement de la,. propriété. ; - il, ..est

prouvé que j de.même, qu'il existe...des

\section{considérables, et que de l'emploi de cess} capitaux naisscnt les richesses qui tendent toujours à s'augmenter, il elt Jémontré que l'inégalité dans les, fortuncs découle -nécessaircment de la proprićré, ; il est prouvé que, de même, qu'il exiṣte des

(b) Image restored with the proposed diffusion filter

considérables, et que de l'emploi de ces capitaux naissent les richesses qui tendent

toujours s'augmenter, il ell dmontré

que l'inǵalité dans les. fortunes découle

nécessairement de la propriété ; ,il est

prouvé que, de.même, qu'il existe des

Fig. 39 Details of the image N001157109-10 [57] before and after restoration with the proposed filter, the Perona-MalikCatté filter and the Weickert diffusion filter. Small extracts of the OCR results obtained on the different document images are also given. 
considérables, et que de l'emploi de ces capitaux naissent les richesses qui tendent toujours à s'augmenter, il eft Jémontré que l'inégalité daus les. fortuncs découle' nécessaircment de la propriḉté; il est prouvé que, de méme, qu'il existe des

\footnotetext{
(a) Image restored with the Perona-Malik-Catté diffusion filter
}

.considérables, et que de l'emploi.de ces .capitaux naissent les richesses qui tendent

?toujours s'augmenter, il eft démontré

nque l'inégalité dans les. fortunes découle'

Jtie'cessaircment .de k.propriété.; -il est

-prouvé que, de même, qu'il existe ..des

\section{considérables, et que de l'emploi de ces} capitaux naisscnt les richesses qui tendcos toujours à s'augmenter, il elt démontré que l'inégalité dans les, fortuncs découle' znécessaircment de la proprićré; ; il est prouvé que, de móme, qu'il cxiste des

(b) Image restored with the Weickert diffusion filter

xpnsidérables, et que de l'emploi de ces

-capitaux naissent les richesses qui tendent

otoujours s'augmenter, il eft démontré

que l'inégalité dans les. fortunes découle

.nécessairement de la propriété.; ,il ejSt

prouvé que, de,môme, qu'il existe des

Fig. 40 Details of the image N001157109-10 [57] before and after restoration with the proposed filter, the Perona-MalikCatté filter and the Weickert diffusion filter. Small extracts of the OCR results obtained on the different document images are also given. 
Aciténeute penff pour condamner,

Toyfelde proye en cicl fevient ofrir,

Apresviotoire a captifs pandonter

Crcmones Mantoue grands matu aura Coufirt.

(a) Original degraded image

A cité neutre penfif pour condamner, I'oylel de proye en ejel fe vient offrir, Apresvietoire a capifs pardoniner, Eremone $\&$ Mantoue grands mallx aupa Coufir.

(b) Image restored with the proposed diffusion filter

A citéneute penfif pour condamner,

Ioyfel de proye en ciel fe vient offrir, Apresvietólie a cajptifs pardonter, Cremone \& Mantoue grands matx aura fouffir.

(c) Image restored with the Perona-Malik-Catté diffusion filter

A ciré neube penff pour condamner, Toypel de proye en eiel fe vient offrir, Apresvietoirea captifs pardonter, cremore \& Mantoue grands malux aupa Soufirit.

(d) Image restored with the Weickert diffusion filter 


\begin{tabular}{|c|c|c|c|c|}
\hline $\begin{array}{c}\text { \% recognition before } \\
\text { restoration }\end{array}$ & $\begin{array}{c}\text { \% recognition after } \\
\text { restoration }\end{array}$ & $\begin{array}{c}\text { Number of } \\
\text { processed characters }\end{array}$ & $\begin{array}{c}\text { Number of errors } \\
\text { before restoration }\end{array}$ & $\begin{array}{c}\text { Number of errors } \\
\text { after restoration }\end{array}$ \\
\hline \hline $92.21 \%$ & $94.46 \%$ & 144847 & 11279 & 8013 \\
\hline
\end{tabular}

Table 11 Impact of the restoration on the recognition rate on 106 color pages of "Gazette of Leyde" [60].

acters degraded by the restoration process (Tab.9).

\subsubsection{Case of Gazettes document images}

Gazettes are the first historical newspapers which have been badly printed on fragile paper. This makes their preservation very difficult. In this respect, numerous national libraries in Europe incite the digitization of these documents. A French project, called "European Gazettes" of the XIII, aims to digitize in order to make the European Gazettes available on the net [59]. For this project, the gazettes are directly digitized in grayscale and with a high resolution. The presence of defects on these supports is mainly explained by the bad quality of document preservation. Figure 42 shows the impact of the restoration which removes most of the noise in the background and smoothes contours. Recognition results of the document image given in figure 42 show a little improvement of $2 \%$ (Tab.9). This little improvement can be explained by the lack of strok discontinuities to restore and the high quality of the digitization process.

Other experiments [60] have been done on a database of 106 color pages of "Gazette of Leyde", scanned at 300 dpi. With 144847 total number of characters, we obtained 11279 errors before restoration and 8013 errors after restoring with our proposed filter. The OCR system had approximately a $94.46 \%$ success rate when detecting restored damaged letters compared to a $92.21 \%$ rate calculated on non-processed characters (tab.11). It is shown that for real document images corrupted by damaged characters, the final restoration images are of improved qualities. We notice that optical character recognition takes great advantage of this improvement for the majority of the tested pages.

\subsubsection{Case of Google books}

Google selects (in priority) non-degraded printed documents and digitizes them directly from the original support instead of the microfilms. Most of the Google book images are scanned in a good quality for a correct recognition rate by an OCR. Few observed degradations are mainly due to the image processing techniques which try to enhance automatically the contrast around textual zones in order to remove ink-bleed through and improve legibility. Figure 43 illustrates the contrast enhancement process from Google which fails when the textual zones are not correctly detected. This happens when the hand of the operator appears in front of the camera or when

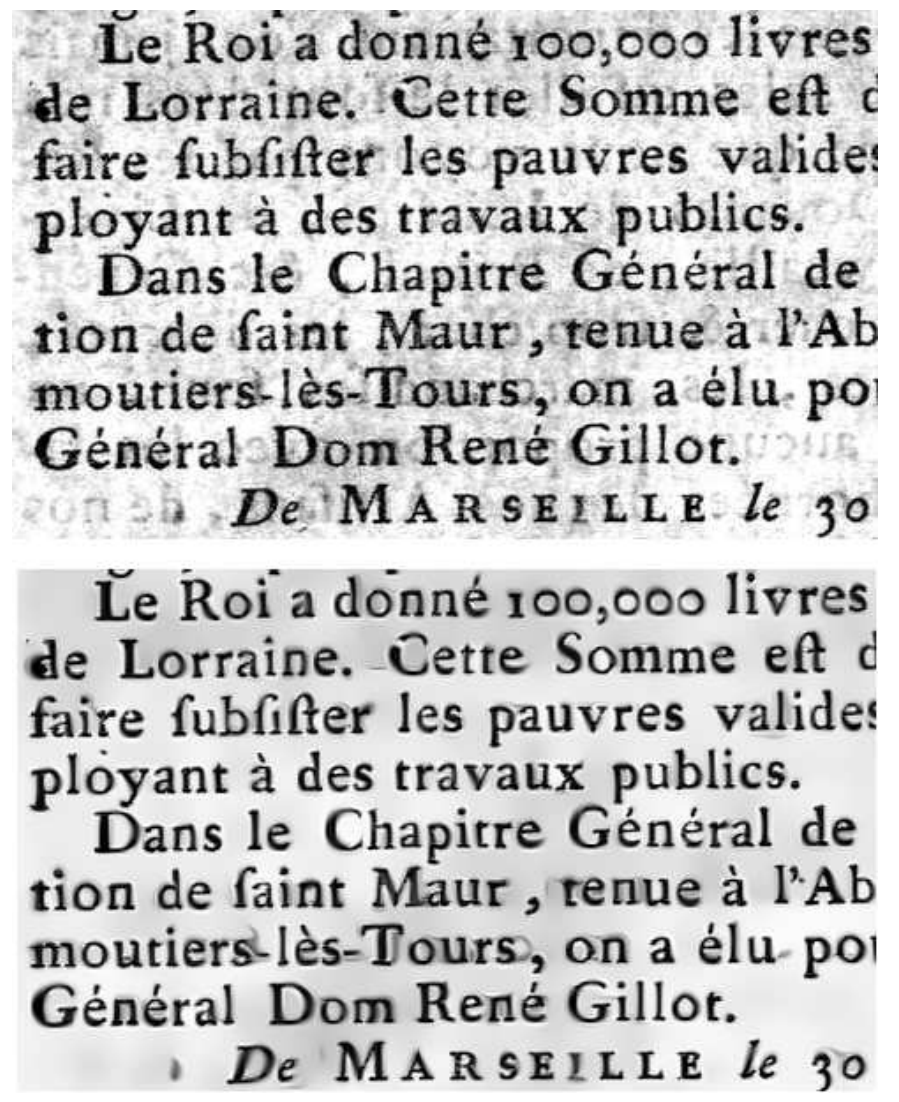

Fig. 42 Details of the image [59] N0070059-TIFF-10-29 before and after restoration respectively from top to down.

the paper background shows too much degradations or are out of the focus. The automatic contrast enhancement remove all the necessary gray scale information to restore images having ink-bleed through degradations. As the application of the diffusion alone is not suited to remove such degradations, we choose the books [61] without such defects and characterized by noise around contours and inside the background.

[61] is a representative historical book (Fig.44.a) from Google, printed in 1690. The layout of this book, which presents large spaces between characters and text lines, makes easier the character segmentation. The total improvement is less than $1 \%$ with $6.18 \%$ of improved characters and $5.22 \%$ of degraded characters (Tab.9). The high rate of characters degraded by the restoration is essentially explained by the numerous characters "e" which become characters "c" after the restoration. For this book, the horizontal line which makes the difference between "e" and "c" is too small and not contrasted enough 
Let the hicredutows make what Hypotbefies they pleare, 'tis unqueftionably true:', 'That the Sucrefion of Gevierations occafion'd the' propidgition of Mantind, and that it ftill ferves continually' for the peopling the Earth we "inhabit. By' thits theans it is come to pafs, thit the 'Fativily of ofie Man which was formerly lodged in a few Terit's, is now fpread over all thie World; and from the abundance of Inbabitants, caite' Coloriles' and Plantations. But foppofé the number of $M \mathrm{M}^{2}$ fhould increare every Year buit ond, by the continual Succeffion of Gener ations, all the Earth mutet neceffarily have alread thihabitants, (If I may one upon another; fin

Eternity.

'Twould be in vain

happen fuch Accil

lick 'Sodieties.

Perfons, fince

and Diforders whic

the rumber of $P$

ftantly increafing,

Fig. 43 Illustration of the automatic contrast enhancement around textual zones detected automatically making ink bleed-through degradations difficult to remove [62] pp.65.

to be preserved during the filtering process(Fig.44.b).

7 Conclusion

We have proven in this work the efficiency of a novel PDE-based approach in enhancing the quality of degraded textual document images and even in reducing their OCR errors. This approach neither requires training nor segmentation steps. It is based on the combination of the Weickert tensor driven diffusion filter and the Perona-Malik scalar diffusion filter and inherits their respective interesting properties: coherence-enhancing and singularities preservation. Properties as such are very essential in processing noisy and broken characters. For instance, the singularities are of twofold as the contours. They improve the legibility of the text as well as the visual quality of an image. Our experiments show that our proposition outperforms Perona-Malik and Weickert filters applied separately. They also illustrate that the scope of this filter is not confined to the processing of textual documents; other kinds of images could also be processed (Fig.45, Fig.46, Fig.47, Fig.48). These claims are corroborated by numerical tests.

As a conclusion, our proposed filter tends to reduce noise, improve useful information and enhance edge continuity than ever was beftow'd ing they fay $26000 l$. terred among the King: he lay till the zoth of Refurrection to anothe he was a fecond time $i$

\section{than cver was beftow'd ing they fay $25000 \%$. tcrred among the King: he lay till the 3 oth of Rcfurrection to anothe he was a fecond time $i$}

Fig. 44 Details of the image [61] before and after restoration respectively from left to right.

simultaneously. These operations are interesting in processing mainly noisy images with damaged characters. Nevertheless, other factors affecting the scanned images should be accounted for as well. In our future work, we intend to investigate PDE-based approaches on other kinds of degradations such as filling gaps in characters or reconstructing larger discontinuities.

\section{References}

1. F. Drira: Towards restoring historic documents degraded over time. In Second IEEE International Conference on Document Image Analysis for Libraries (DIAL'2006), Lyon, France. pp. 350-357. ISBN 0-7695-2531-4. (2006).

2. M. Cannon, J. Hochberg, P. Kelly: Quality Assessment and Restoration of Typewritten Document Images. International Journal of Document Analysis and Recognition, Vol.2, pp. 80-89 (1999).

3. V. Aurich, J. Weule: Non-linear gaussian filters performing edge preserving diffusion. In Proceedings of the DAGM Symposium, Springer-Verlag, pp.538-545 (1995).

4. L. Rudin, S. Osher : Nonlinear total variation based noise removal algorithms, Physica D: Nonlinear Phenomena, Vol. 60, pp.259-268 (1992).

5. A. Buades, B. Coll, J. Morel. A non local algorithm for image denoising. In Proceedings of International Con- 

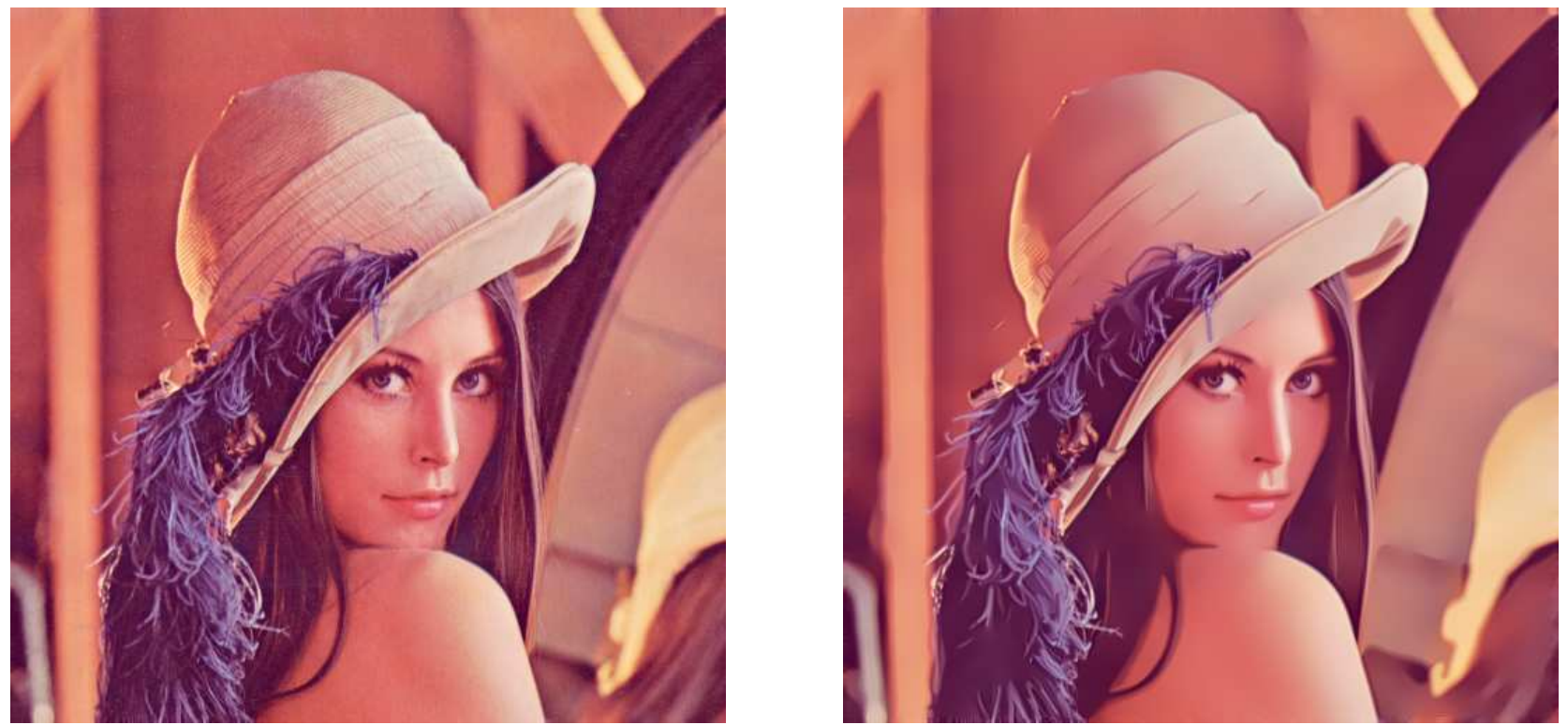

Fig. 45 Restoration of a natural image (classical image of Lena) with the proposed filter; respectively from left to right, details before and after restoration.
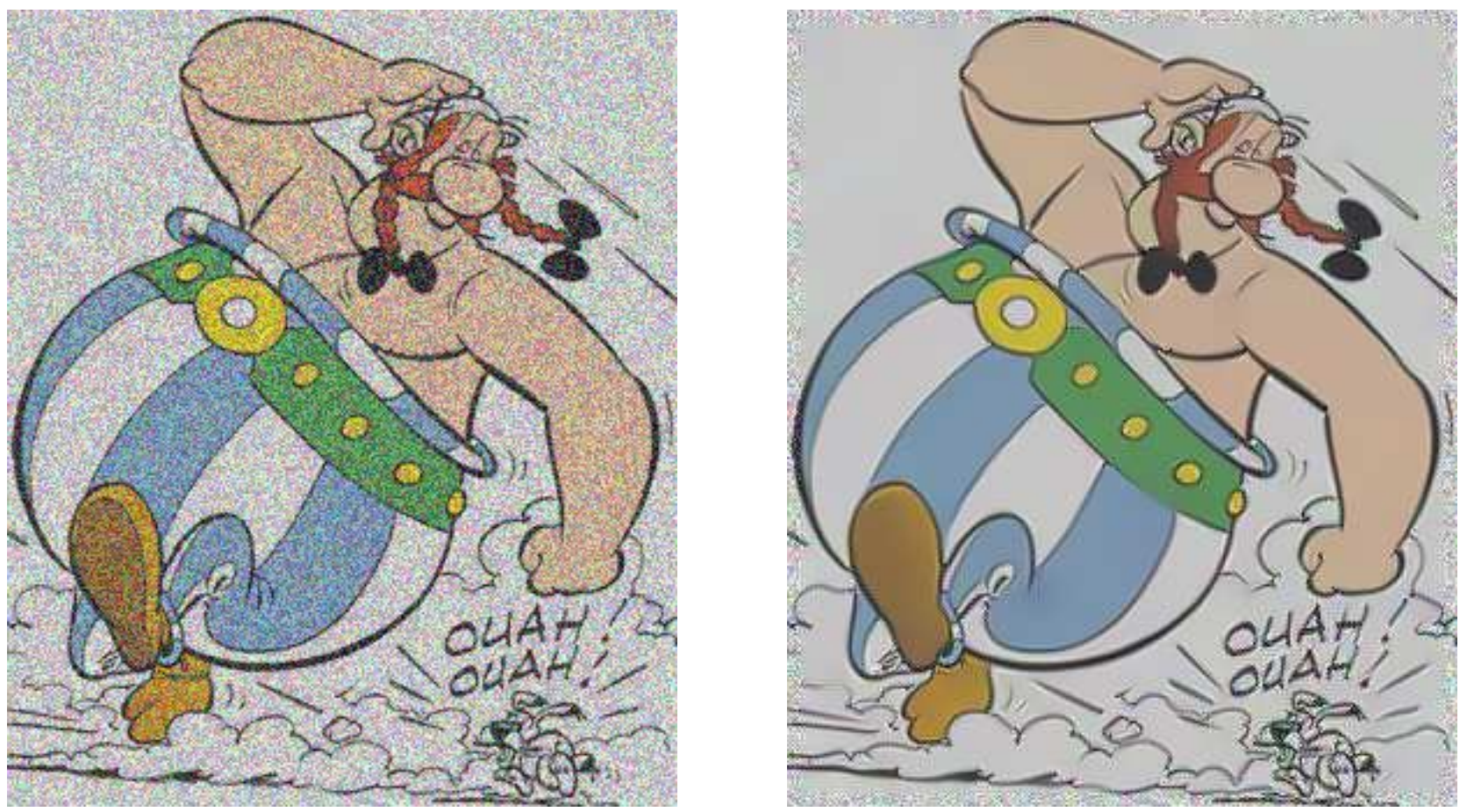

Fig. 46 From left to right respectively : Original image and its restored version with our proposed diffusion filter.

ference on Computer Vision and Pattern Recognition (CVPR), Vol.2, pp. 60-65 (2005).

6. A. Buades, B. Coll, J. Morel. A review of image denoising algorithms, with a new one. SIAM Interdisciplinary Journal:Multiscale Modeling and Simulation, 4(2):290$530(2005)$.

7. A. Dauwe, B. Goossens, H. Luong, W. Philips. A fast non-local image denoising algorithm. In Proceedings of SPIE Electronic Imaging, volume 6812 (2008).

8. P. Coup, P. Yger, S. Prima, P. Hellier, C. Kervrann, C. Barillot. An optimized blockwise nonlocal means denois- ing filter for 3-D magnetic resonance images. IEEE Transactions on Medical Imaging, 27(4):425-441 (2008).

9. P. Chatterjee, P. Milanfar. A generalization of non-local means via kernel regression. In Proceedings of SPIE Electronic Imaging, volume 6814 (2008).

10. S. Zimmer, S. Didas, J. Weickert : A rotationally invariant block matching strategy improving image denoising with non-local means. In Proceedings of International Workshop on Local andNon-Local Approximation in Image Processing, pp. 135-142 (2008). 

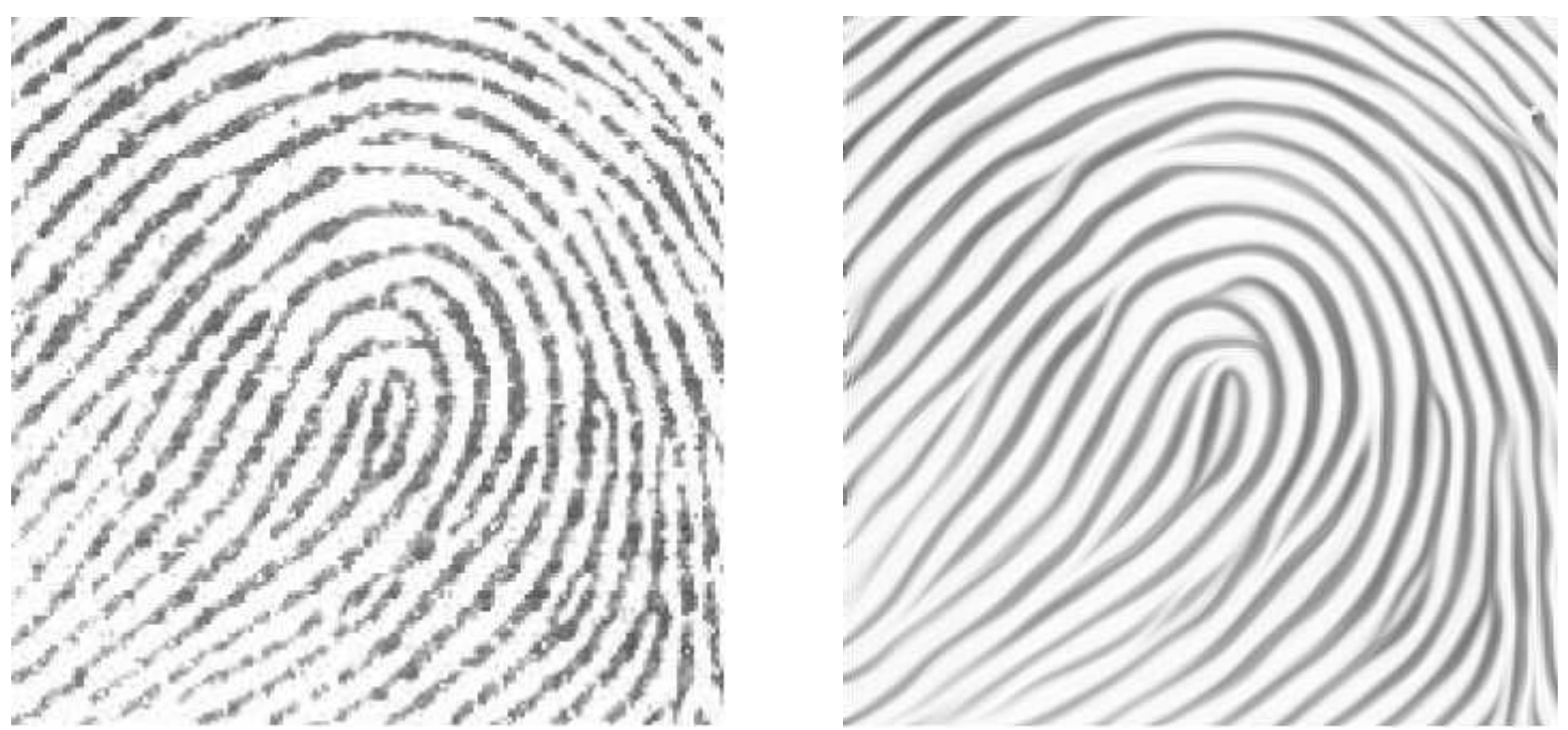

Fig. 47 From left to right respectively : Original image and its restored version with our proposed diffusion filter.
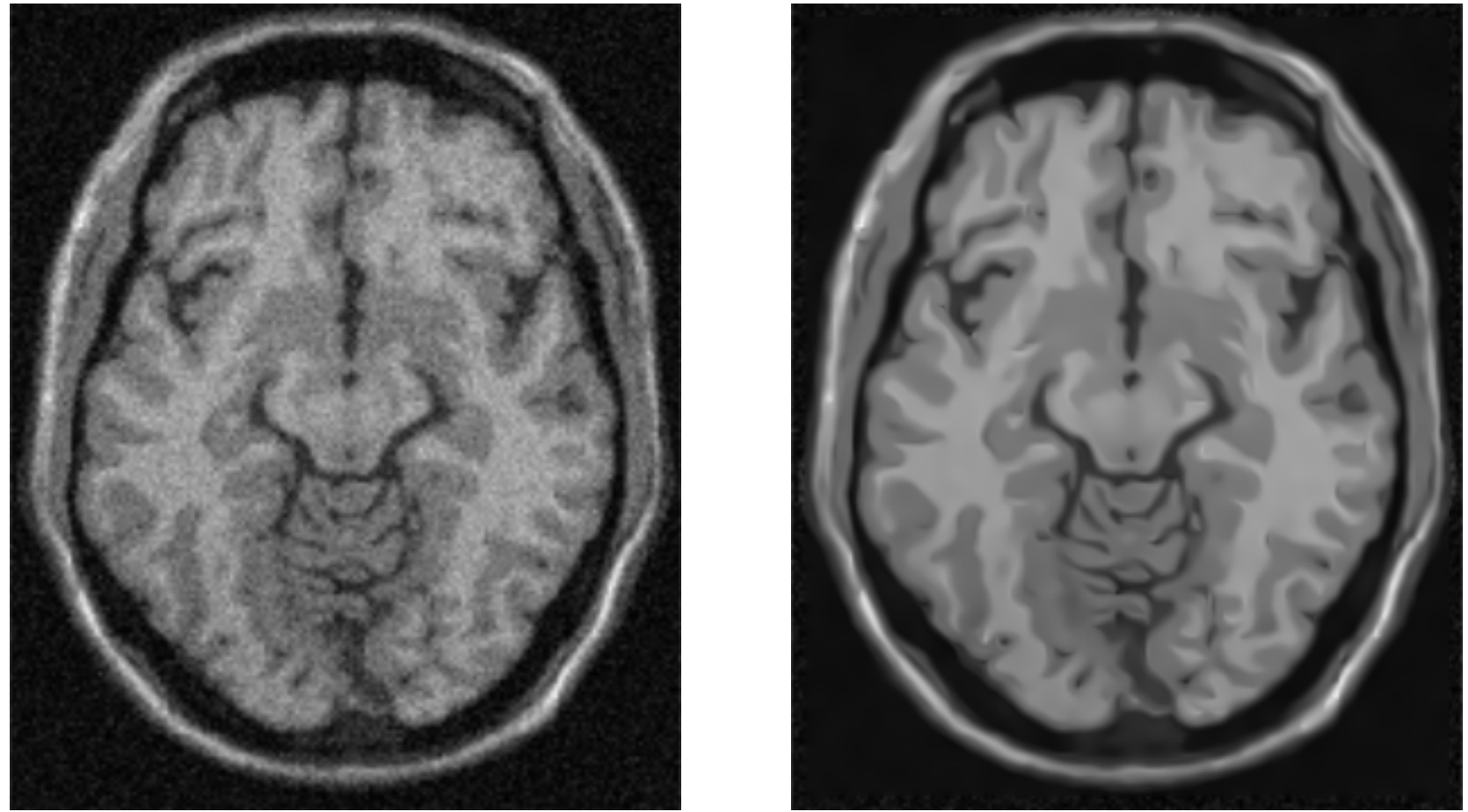

Fig. 48 From left to right respectively : Original medical image and its restored version with our proposed diffusion filter.

11. M. Aharon, M. Elad, A. Bruckstein. KSVD: An algorithm for designing overcomplete dictionaries for sparse representation. IEEE Transactions on Signal Processing, 54(11):4311-4322 (2006).

12. R. Nakagaki, A. Katsaggelos. A VQ-based blind image restoration algorithm. IEEE Transactions on Image Processing, 12(9):1044-1053 (2003).

13. S. Roth and M. J. Black: Fields of Experts, In International Journal of Computer Vision (IJCV), 82(2):205-229 (2009).
14. J. Mairal, M. Elad, G. Sapiro, Sparse representation for color image restoration, IEEE Transactions on Image Processing, Vol. 17, pp. 53-69 (2008).

15. H. Nishida. Restoring high-resolution binary images for text enhancement. In ICIP, pp. 506-509 (2005).

16. Q. Zheng and T. Kanungo. Morphological degradation models and their use in document image restoration.. in ICIP'01, pp. I: 193-196 (2001).

17. J. Liang and R. M. Haralick. Document image restoration using binary morphological filters. In SPIE'96, Vol. 2660, pp. 274-285 (1996). 
18. F. Sattar and D. Tay. Enhancement of document images using multiresolution and fuzzy logic techniques. Signal Processing Letters, Vol. 6, pp. 249-252, (1999).

19. Z. Shi and V. Govindaraju. Historical Document Image Enhancement Using Background Light Intensity Normalization. 17th International Conference on Pattern Recognition (ICPR'04), Vol. 1, pp. 473-476 (2004).

20. P. Sarkar, H. Baird, and X. Zhang. Training on severely degraded text-line images. In Proceedings of the International Conference on Document Analysis and Recognition, pp. 38-43 (2003).

21. A. Tonazzini, S. Vezzosi, and L. Bedini. Analysis and recognition of highly degraded printed characters. International Journal on Document Analysis and Recognition, 6:236-247 (2004).

22. H. Luong and W. Philips. Non-Local Text Image Reconstruction. Ninth International Conference on Document Analysis and Recognition (ICDAR'2007), Vol.1, pp.546550 (2007).

23. J. Banerjee, A. M. Namboodiri and C. V. Jawahar. Contextual restoration of severely degraded document images. In Proceeding of IEEE International Conference on CVPR, pp 517-524 (2009).

24. A. Tonazzini, E. Salerno, L. Bedini : Fast Correction of Bleed-Through Distortion in Grayscale Documents by a Blind Source Separation Technique, International Journal of Document Analysis and Recognition, vol.10, pp.1725 (2007).

25. C. Wolf:Document Ink bleed-through removal with two hidden Markov random fields and a single observation field, IEEE Transactions on Pattern Analysis and Machine Intelligence (PAMI),no.32, pp.431-447 (2010).

26. R. F. Moghaddam and M. Cheriet : RSLDI: Restoration of single-sided low-quality document images, Pattern Recognition, Special Issue on Handwriting Recognition, no. 42, pp. 3355-3364 (2009).

27. I. Nwogu, Z. Shi and V. Govindaraju. PDE-Based Enhancement of Low Quality Documents. In Proceedings of the Ninth international Conference on Document Analysis and Recognition - Vol.01, pp.541-545 (2007).

28. F. Drira, F. Lebourgeois, H. Emptoz: Restoring Ink Bleed-Through Degraded Document Images Using a Recursive Unsupervised Classification Technique. In DAS'2006, LNCS 3872. Nelson, New Zealand, pp. 38-49. (2006).

29. F Drira, F Lebourgeois, H. Emptoz : A Modified Mean Shift Algorithm For Efficient Document Image Restoration, In Signal processing for image enhancement and multimedia processing Ed. Springer Verlag, ISBN 978-0-387-72499-7, on-line copy http://www.springerlink.com/content/978-0-387-724997 (2008).

30. A. Ledda A. Ledda, H.Q. Luong, W. Philips, V. De Witte and E.E. Kerre. Greyscale Image Interpolation using Mathematical Morphology. In Proceedings of ACIVS (LNCS 4179), pp. 78-90 (2006).

31. J. D. Hobby, H. S. Baird, Q. Zheng, T. Kanungo: Morphological degradation models and their use in document image restoration, ICIP'01, Greece, pp. 193-196. (2001).

32. J. D. Hobby, H.S. Baird: Degraded Character Image Restoration. In Proc. of Document Analysis and Information Retrieval, Las Vegas. (1996).

33. H. S. Baird : State of the Art of Document Image Degradation Modeling. In Proceedings of 4th IAPR International Workshop on Document Analysis Systems, vol. 1, Rio de Janeiro, Brazil (2000).

34. J. D. Hobby, T.K.Ho: Enhancing Degraded Document Images via Bitmap Clustering and Averaging. In Proceeding of the fourth International Conference on Document Analysis and Recognition, ICDAR'97, Germany, pp. 394-400 (1997).
35. A. Whichello, H. Yan. Linking Broken Character Borders with Variable Sized Masks to Improve Recognition, Pattern Recognition, vol. 29, no. 8, pp. 1429-1435 (1996). 36. http://www.impact-project.eu/

37. L. Alvarez, F. Guichard, P. L. Lions, J. M. Morel: Axioms and Fundamental Equations of Images Processing, Archive for Rational Mechanics and Analysis, 123 (3), pp. 199-257 (1993).

38. J. Weickert : a review of nonlinear diffusion filtering, In Scale-Space Theory in Computer Vision, volume 1252 of Lecture Notes in Computer Science, Utrecht, the Netherlands (1997).

39. J. Weickert: Anisotropic Diffusion in Image Processing, Stuttgart, teubner-Verlag (1998).

40. L. Alvarez, F. Guichard, P. L. Lions, J. M. Morel: Axioms and Fundamental Equations of Images Processing, Archive for Rational Mechanics and Analysis, 123 (3), pp 199-257 (1993).

41. A. Tikhonov, V. Arsenin: Solution of Ill-Posed Problems, Winston and Wiley (1977).

42. P. Perona, J. Malik : Scale-Space and Edge Detection Using Anisotropic Diffusion, In IEEE Trans. Pattern. Analysis and Machine Intelligence, pp. 629-639 (1990).

43. P.Perona, T. Shiota, J.Malik : Anisotropic Diffusion. In Bart M. Ter Haar Romeny Editor, Geometry-driven diffusion in computer vision. Dordrecht : Kluwer Academic, 1994, pp. 229-254 (1994).

44. F. Catté, J. M. Morel, P. L. Lions, T. Coll : Image Selective smoothing and edge detection by nonlinear diffusion, In SIAM J. Numer. Anal., 29:182-193 (1992).

45. J. Weickert: Scale-space properties of nonlinear diffusion filtering with a diffusion tensor. Report No. 110. Laboratory of Technomathematics, University of Kaiserslautern, Germany (1994).

46. J. Weickert : Anisotropic Diffusion in Image Processing, Stuttgart, teubner-Verlag (1998).

47. J. Weickert: Coherence-enhancing diffusion of colour images. In the 7th National Symposium on Pattern Recognition and Image Analysis, Barcelona, Spain(1997).

48. N. Sochen, R. Kimmel, R. Malladi: A geometrical framework for low level vision. IEEE Transaction on Image Processing, Special Issue on PDE based Image Processing, 7(3):310.318 (1998).

49. R. Kimmel, R. Malladi, N. Sochen : Images as embedded maps and minimal surfaces: movies, color, texture, and volumetric medical images. International Journal of Computer Vision, 39(2):111.129 (2000).

50. D. Tschumperlé, R. Deriche : Vector-Valued image regularisation with PDE's: A common framework for different applications. IEEE transactions on Pattern Analysis and Machine Intelligence, vol.27, No 4 (2005).

51. H. S. Baird: The state of the art of document image degradation modeling, in book, In Digital Document Processing Advances in Pattern Recognition, DOI: 10.1007/978-1-84628-726-8-12, pp. 261-279 (2007).

52. E. H. B. Smith and T. Andersen :Text Degradations and OCR Training,In International Conference on Document Analysis and Recognition 2005, Seoul, Korea, pp. 834-838 (2005).

53. C. Hal, E. H. B. Smith : Human Image Preference and Document Degradation Models, In International Conference on Document Analysis and Recognition 2007 (2007).

54. Les prophéties de M. Michel Nostradamus, Nostradamus, Bibliothèque nationale de France, 1589, http://gallica2.bnf.fr/ark:/12148/bpt6k700592

55. LE CONSTITUTIONNEL, journal politique et littéraire, exemplaire du Mardi 31 octobre 1815, Paris, ark:/12148/cb327475869/date , ISSN 17706165, Bibliothque nationale de France, http://gallica.bnf.fr/ark:/12148/bpt6k648239f.pleinepage.langFR 
56. Le bourgeois gentilhomme, comédie-balet faite Chambort, pour le divertissement du Roy, Molière, 1671, Bibliothèque nationale de France, Rés. p-Yf-56,

http://gallica2.bnf.fr/ark:/12148/bpt6k70212z

57. Discours sur les avantages ou les désavantages qui résultent pour l'Europe de la découverte de l'Amérique, objet du prix proposé par M. l'abbé Raynal, FranoisJean Chastellux, 1787, Bibliothèque nationale de France http://gallica2.bnf.fr/ark:/12148/bpt6k1157109

58. http://gazettes18e.ish-lyon.cnrs.fr

59. Courrier d'Avignon, du 4 juillet 1775, http://gazettes18e.ish-lyon.cnrs.fr/

60. F Drira, F Lebourgeois, H. Emptoz : Document images restoration by a new tensor based diffusion process: Application to the recognition of old printed documents, In 10th International Conference on Document Analysis and Recognition (ICDAR), IEEE ed. Spain. pp.321-325 (2009).

61. An epitomy of English history, Thomas May, Printed for N. Boddington at the Golden Ball in Duck lane, 1690, University of Michigan, digitized 5 june 2007, http://books.google.fr/books?id=kSE2AAAAMAAJ

62. A vindication of the truth of Christian religion: against the objections of all modern opposers,Volume 1, Jacques Abbadie, 1694, New York Public Library, digitized 1st december 2005. http:/ / books.google.fr $/$ books?id $=5 \mathrm{crJcHvTpZcC}$ 